\title{
Lung-resident memory B cells protect against bacterial pneumonia
}

\author{
Kimberly A. Barker, ${ }^{1,2}$ Neelou S. Etesami, ${ }^{1,2}$ Anukul T. Shenoy, ${ }^{1}$ Emad I. Arafa, ${ }^{1,3}$ Carolina Lyon de Ana, ${ }^{1,2}$ Nicole M.S. Smith, ${ }^{1,4}$ \\ Ian M.C. Martin, ${ }^{1}$ Wesley N. Goltry, ${ }^{1}$ Alexander M.S. Barron, ${ }^{2}$ Jeffrey L. Browning, ${ }^{2,3}$ Hasmeena Kathuria, ${ }^{1,3}$ Anna C. Belkina, ${ }^{1,4,5}$ \\ Antoine Guillon, ${ }^{1,6}$ Xuemei Zhong, ${ }^{3}$ Nicholas A. Crossland, ${ }^{4}$ Matthew R. Jones, ${ }^{1,3}$ Lee J. Quinton, ${ }^{1,2,3,4}$ and Joseph P. Mizgerd ${ }^{1,2,3,7}$ \\ ${ }^{1}$ Pulmonary Center, ${ }^{2}$ Department of Microbiology, ${ }^{3}$ Department of Medicine, ${ }^{4}$ Department of Pathology and Laboratory Medicine, and ${ }^{5}$ Flow Cytometry Core Facility, Boston University School of Medicine, \\ Boston, Massachusetts, USA. 'Centre Hospitalier Régional Universitaire de (CHRU) de Tours, Service de Médecine Intensive Réanimation, INSERM, Centre d’Etude des Pathologies Respiratoires (CEPR), \\ UMR 1100, University of Tours, Tours, France. ’Department of Biochemistry, Boston University School of Medicine, Boston, Massachusetts, USA.
}

\begin{abstract}
Lung-resident memory B cells (BRM cells) are elicited after influenza infections of mice, but connections to other pathogens and hosts - as well as their functional significance - have yet to be determined. We postulate that BRM cells are core components of lung immunity. To test this, we examined whether lung BRM cells are elicited by the respiratory pathogen pneumococcus, are present in humans, and are important in pneumonia defense. Lungs of mice that had recovered from pneumococcal infections did not contain organized tertiary lymphoid organs, but did have plasma cells and noncirculating memory B cells. The latter expressed distinctive surface markers (including CD69, PD-L2, CD80, and CD73) and were poised to secrete antibodies upon stimulation. Human lungs also contained B cells with a resident memory phenotype. In mice recovered from pneumococcal pneumonia, depletion of PD-L2+ B cells, including lung BRM cells, diminished bacterial clearance and the level of pneumococcus-reactive antibodies in the lung. These data define lung BRM cells as a common feature of pathogenexperienced lungs and provide direct evidence of a role for these cells in pulmonary antibacterial immunity.
\end{abstract}

\section{Introduction}

Humans evolved alongside a vast array of microbial organisms and environmental antigens. Consequently, our immune system is highly adaptable, undergoing long-term systemic and mucosal remodeling following exposure to commonly encountered external stimuli (1). Traditional specific pathogen-free (SPF) laboratory mice immunologically resemble human infants and lack immune features observed in healthy adults, including resident lymphocytes in nonlymphoid tissues, but gain these more humanlike immune features upon pathogen exposure $(1,2)$.

Tissue-resident immune cells comprise a group of noncirculating leukocytes that act as a frontline barrier of defense, especially at mucosal sites of constant environmental antigen exposure such as the lung. Although multiple immune cell types, including innate immune cells, may be resident, the long-term protective potential of adaptive memory cells makes resident memory lymphocytes particularly intriguing. Resident memory T cells (TRM cells) populate mucosal surfaces in response to various bacterial and viral pathogens, and provide rapid local protection against reinfection (3). In the lung, TRM cells can be maintained in niches independently of organized induced bronchus-associated lymphoid tissue (iBALT, ref. 4). Although TRM cells have been extensively studied over the last 2 decades, it remains uncertain whether complementary resident memory B cells (BRM cells) are a common feature at mucosal sites.

Conflict of interest: The authors have declared that no conflict of interest exists. Copyright: () 2021, American Society for Clinical Investigation.

Submitted: June 29, 2020; Accepted: April 14, 2021; Published: June 1, 2021

Reference information: J Clin Invest. 2021;131(11):e141810.

https://doi.org/10.1172/JCl141810.
Memory B cells (MBCs) located in mucosal tissues play important roles in mice and humans, but generally are found in organized lymphoid structures such as gut-associated lymphoid tissue (5) and human tonsils/adenoids (6). In the female mouse reproductive tract, herpes simplex virus immunization generates local TRM cells without concurrent BRM cell formation, indicating that the presence of mucosal TRM cells does not always correlate with establishment of a BRM cell pool (7). Lung BRM cells and lung plasma cells are elicited by influenza infections in mice (8-10). These B cells do not recirculate (8) and provide enhanced protection when adoptively transferred compared with splenic influenza-specific MBCs (10). However, the detailed location of these BRM cells in the lung has not to our knowledge been described. Notably, influenza generates long-lasting iBALT in mice (11). Like other organized lymphoid structures, iBALT has been shown to support MBCs and plasma cells but is not a typical feature of healthy adult human lungs $(12,13)$. Lungs of rhesus macaques recovered from asymptomatic H1N1 influenza infection are enriched for TRM but not BRM cells, a finding attributed to the likely lack of iBALT in such low-virulence infections (14). Thus, whether lung BRM and plasma cells are unique to iBALTcontaining mouse lungs after influenza or are a common feature of the lung adaptive immune cell landscape remains to be determined.

Both viral and bacterial lung infections impart a large burden of disease globally and in the United States, especially among children and the elderly, for whom pneumonia leads to more deaths than any other infectious disease $(15,16)$. Streptococcus pneumoniae (pneumococcus), comprising nearly 100 serotypes defined by polysaccharide capsule, represents the most common bacterial cause of community-acquired pneumonia (17). Nearly all children, even those given pneumococcal vaccination, are colonized or infected multiple times 
with pneumococcus before the age of 2 years (18). These natural exposures generate serotype-independent (heterotypic) immune protection (19). Heterotypic antibodies, long-lived plasma cells (LLPCs), and lung TRM cells contribute to naturally acquired immunity to pneumococcus (20), but a role for MBCs has yet to be investigated. Given that virus-elicited MBCs can harbor cross-reactive specificities against mutated viral strains $(21,22)$, we hypothesized that MBCs may play a similar role in immunity against multiple pneumococcal serotypes. Therefore, we undertook this study to address the fundamental gaps in knowledge regarding the existence of lung-resident $\mathrm{B}$ cells outside the setting of influenza-recovered mouse lungs and the contribution of these cells to antibacterial lung immunity.

\section{Results}

Effects of resolved pneumococcal pneumonias on circulating cells and antibodies. Resolution of pneumococcal pneumonias in mice provides serotype-independent lung protection against subsequent pneumonia conferred in part by $\mathrm{CD} 4^{+} \mathrm{TRM}$ cells and remodeled alveolar macrophages (23-25). We suspected that additional immune changes elicited by pneumococcal exposures remained to be identified. The remodeled immunity in SPF mice exposed to pathogen-rich mice sourced from a pet store was defined by a profound change in circulating blood leukocytes evident in their transcriptomes (1). To investigate whether respiratory infections with $S$. pneumoniae elicited similar systemic changes, we infected mice in the lung with pneumococcal serotype 19F (Sp19F) twice with a 1-week interval (Figure 1A). In the majority of experiments (unless indicated otherwise in the legends), these instillations were given intratracheally (i.t.) and directed into only the animal's left lung lobe. Such infections are self-limiting, and animals fully recover without the aid of antibiotics or supportive treatment (24). Control mice were concurrently given saline instillations. Infected and control mice were rested for at least 4 weeks. Previously infected mice were referred to as "experienced," while control mice were referred to as "naive." As described above, experienced mice have serotype-independent (heterotypic) immune protection, as evidenced by their ability to clear a serotypemismatched challenge infection with $\mathrm{Sp} 3$, which grows uncontrollably in naive mice (Figure 1B). Unlike the radically transformed blood transcriptomes of so-called dirty mice compared with naive laboratory mice (1), whole blood transcriptomes of uninfected experienced and naive mice in our model were essentially identical (Figure 1, C and D). Only 4 genes were differentially expressed $(\mathrm{FDR}<0.05)$ at the mRNA level between naive and experienced mice (Lpp, Pmaip1, $N t 5 c$, and Arfgap2), and none of these exhibited a fold change greater than $1.36(0.76,1.36,1.22$, and 1.23 , respectively). Thus, while prior studies revealed new cells and altered resident cell phenotypes in the lungs of mice recovered from such Sp19F infections (23-25), the circulating cell transcriptomes were unchanged.

Memory B cells in pneumococcus-experienced lungs. The lack of systemic transcriptomic differences between naive and experienced mice led us to focus on unexplored cell types in the lung itself that may be contributing to improved immunity in experienced mice. Depletion of $\mathrm{CD}^{+}{ }^{+} \mathrm{TRM}$ cells before Sp3 challenge only partially abrogates lung protection in experienced mice (23). Additionally, in mice initially given a single-lobe infection, protection is limited to the exposed lung lobe and is not seen in the contralateral lobes, indicating that systemic factors such as circulating antibodies are not sufficient (23). Based on these findings and the recent descriptions of lung BRM cells in mice recovered from influenza $(8,10$, 22), we hypothesized that MBCs may be found in pneumococcusexperienced lungs. At 4 weeks after the last Sp19F exposure, lungs of experienced and naive mice are histologically normal and do not contain visible iBALT structures by H\&E staining (23). To localize B cells, we used immunofluorescence microscopy to illuminate $\mathrm{B}^{2} 2 \mathrm{O}^{+}$ and $\mathrm{CD} 4^{+}$cells in frozen sections from naive and experienced lungs (Figure 2, A and B). B220 ${ }^{+}$cells were observed in experienced but not naive lungs, typically near the conducting airways, in small aggregates of cells as well as loosely dispersed, in some cases in association with $\mathrm{CD}^{+}$cells (Figure 2, A and B). We complemented these analyses with $H \& E$ staining in formalin-fixed sections from naive and experienced lungs and with immunohistochemical analysis of serial sections illuminating $\mathrm{CD} 19^{+}$along with $\mathrm{CD}^{+}$cells. Small mixed leukocyte aggregates were discernible within bronchovascular bundles of experienced (Figure 2, D-F) but not naive (Figure 2C) mice. Most of these cells were either $\mathrm{CD} 19^{+}$or $\mathrm{CD} 4^{+}$(Figure 2, E and F). The $\mathrm{CD}_{19}{ }^{+}$cells accumulated in the loose interstitial tissue connecting the conducting airways with the pulmonary arteries, closer to the airways than the arteries (Figure 2, E and F). Thus, B cells in the lungs of experienced mice were in the connective tissue of distal bronchovascular bundles, proximal to bronchiolar airways, often but not always in association with $\mathrm{CD}^{+} \mathrm{T}$ cells. To assess whether the high endothelial venules (HEVs) characteristic of tertiary lymphoid organs were present in experienced mouse lungs, we stained lung sections for CD34 and peripheral node addressin (PNAd), which costain HEVs in well-organized lymphoid structures such as lymph nodes (Supplemental Figure 1A; supplemental material available online with this article; https://doi.org/10.1172/JCI141810DS1) and lung iBALT (12). Although the lung sections stained brightly for the vascular endothelial marker CD34, we did not observe PNAd staining, indicating that HEVs were not present in these lung sections (Supplemental Figure 1, B-D).

To phenotype these lung B cells, we used flow cytometry with an i.v. anti-CD45 stain to distinguish circulating from lung leukocytes. A population of $\mathrm{CD} 19^{+}$cells protected from the i.v. stain, defined as extravascular (EV) B cells, was observed in lungs of experienced but not naive mice (Figure 2G; gating scheme, Supplemental Figure 2A). The EV B cells in experienced mice were predominantly $\operatorname{IgD}^{-}$, while the intravascular (IV) B cells positive for the i.v. stain in the same mice were largely $\operatorname{IgD}^{+}$(Figure 2, $\mathrm{H}$ and I). Additionally, the percentage of EV lung $\operatorname{IgD}^{-} \mathrm{B}$ cells, but not $\mathrm{EV} \mathrm{IgD}^{+} \mathrm{B}$ cells, was significantly increased by experience, and there was no effect of experience on the IV B cells in these lung digests (Figure 2I). These data suggest that the lung EV B cell compartment after pneumococcal exposures was enriched for non-naive B cells, which we hypothesized consisted largely of memory cells. Consistent with this hypothesis, the number of IgD ${ }^{-}$EV B cells in experienced lungs remained constant for as long as 12 weeks after the last Sp19F infection before declining by 6 months after exposure, when the significant difference in lung $\mathrm{B}$ cell numbers between experienced and naive mice was lost (Figure 2J). Moreover, all the $\mathrm{EV} \mathrm{IgD}^{-} \mathrm{B}$ cells in experienced mice were $\mathrm{CD}_{38^{+}}$, an $\mathrm{MBC}$ phenotype in mice (Figure 2K; gating scheme, Supplemental Figure 2B; and ref. 26). Together, these results indicate that repeated pneumococcal exposures in mice elicit MBCs in the absence of tertiary lymphoid organs. These MBCs localize to 
A
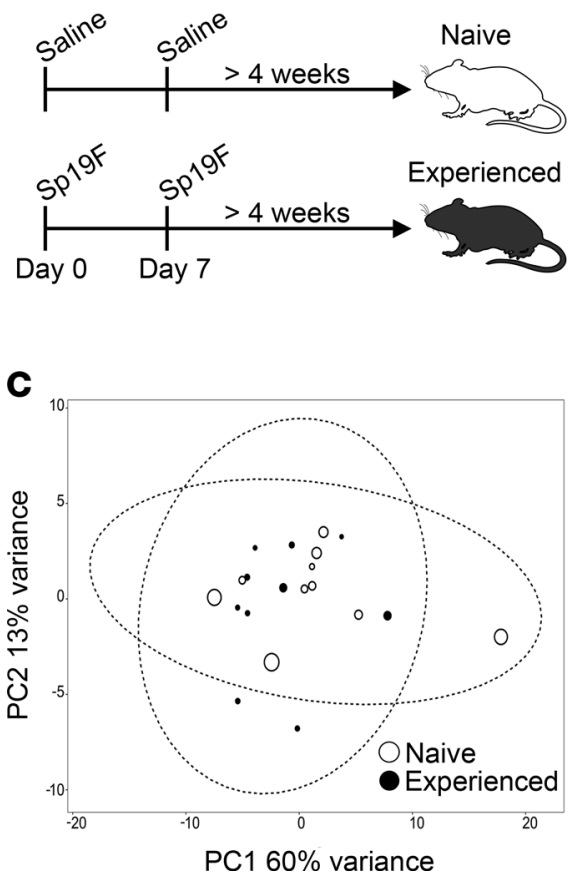

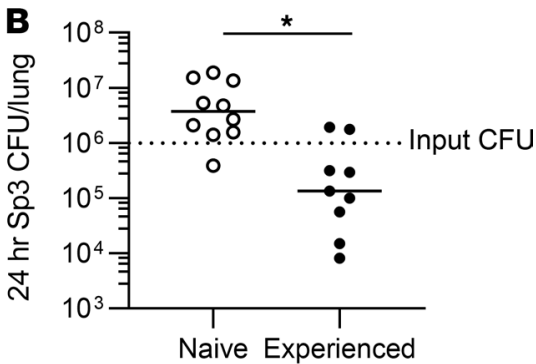

D

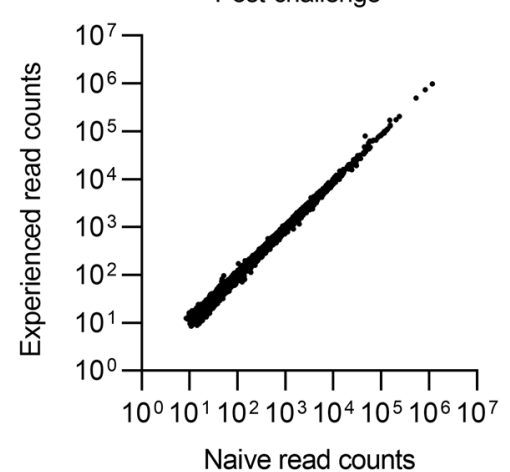

Figure 1. Pneumococcal exposures provide lung protection without extensive changes to the blood transcriptome. (A) B6 mice were exposed to i.t. or i.n. pneumococcus (Sp19F) or saline in the left lung lobe twice with a 1-week interval, then allowed to recover for at least 4 weeks, at which point they were referred to as "experienced" or "naive," respectively. (B) Experienced and naive mice were challenged with i.t. pneumococcus ( $\mathrm{Sp} 3$ ) for 24 hours before lung bacterial burdens were assessed (Mann-Whitney $U$ test, ${ }^{*} P=0.0006$ ). Principal component analysis (C) and read counts (D) from RNA-Seq of whole blood collected from the same naive and experienced mice as in $\mathbf{B}$ prior to $\mathrm{Sp} 3$ challenge. Data point size in $\mathbf{C}$ is proportional to the 24-hour lung Sp3 CFU of each mouse in $\mathbf{B}$. Each point in $\mathbf{D}$ represents 1 gene.

bronchovascular bundles, with or without associated $\mathrm{CD} 4^{+} \mathrm{T}$ cells, in the loose connective tissue proximal to bronchioles.

Plasma cells in lungs and bone marrow of experienced mice. In addition to eliciting quiescent MBCs, recovery from infection can result in LLPCs constitutively producing antibodies. Consistent with prior studies (27), the mice recovered from Sp19F infections had heterotypic antipneumococcal antibodies in the blood (Figure 3A). We hypothesized that these antibodies derived at least partially from LLPCs in experienced mouse bone marrow. Pneumococcus-specific IgG and IgA antibodies were detected in supernatant of cultured unstimulated bone marrow cells from experienced but not naive mice (Figure 3B), confirming the establishment of bone marrow plasma cells after lung infection by pneumococcus. After influenza infections, antibody-secreting plasma cells can be found within the lung tissue $(8,9)$. To test whether pneumococcal exposures establish such cells in the lung, we cultured unstimulated EV lung cells on ELISpot plates. Spots representing plasma cells secreting IgA or IgG were detected only in wells containing samples from experienced mouse lungs (Figure $3, \mathrm{C}$ and D). EV lung cells bearing a $\mathrm{CD} 138^{+} \mathrm{CD} 38^{\text {lo }}$ plasma cell phenotype were enriched in lung cell digests from experienced versus naive mice (Figure 3, E and F; gating, Supplemental Figure 3). Plasma cell identity was confirmed by expression of the transcription factor Blimp-1 (28) in the $\mathrm{CD} 138^{+}$but not the $\mathrm{CD} 138^{-}$cell population (Figure $3 \mathrm{G})$. While the $\mathrm{CD} 38^{+} \mathrm{CD} 138^{-}$cell population in the lung included $\mathrm{B}$ cells expressing $\mathrm{B} 220$ and $\mathrm{CD} 19$, the $\mathrm{CD} 38^{-} \mathrm{CD} 138^{+}$population did not (Supplemental Figure 3), further supporting the identification of the former as LLPCs (29). The presence of plasma cells within the lung tissue prompted investigation of pneumococcus-specific antibodies in the air spaces of experienced lungs. ELISA analysis of bronchoalveolar lavage fluid (BALF) showed that heterotypic antipneumococcal antibodies were recovered from the uninfected air spaces of experienced but not naive mice (Figure 3H). Together, these studies indicate that respiratory pneumococcal infections elicit LLPCs secreting heterotypic antipneumococcal antibodies in the bone marrow and lung, complementing the accumulation of MBCs in the lung.

Resident phenotype of lung MBCs after pneumococcal exposures. The lack of i.v. CD 45 staining of lung B cells in experienced mice indicated that these cells were excluded from the circulation at the time of assay. We hypothesized that the lung MBCs represent a resident immune cell pool, and considered whether they bore surface markers similar to those of lung TRM cells $\left(\mathrm{CD} 69^{+} \mathrm{CD} 11 \mathrm{a}^{\text {bright }} \mathrm{C}\right.$ D62 $\left.{ }^{\text {lo }} \mathrm{CD} 44^{+}\right)$(23). Consistent with prior reports on lung BRM cells $(8,10)$, many of the EV B cells in experienced mice expressed the lymphocyte residence marker CD69 and the integrin CD11a (Figure 4A). Compared with IV lung B cells, EV lung B cells had lower expression of the lymphoid organ homing molecule CD62L and higher expression of the glycoprotein CD44 (Figure 4A). The similarity in surface marker profiles between MBCs in pneumococcus-experienced lungs and lung TRM cells reported in many experimental systems indicates that the MBCs observed here are likely to be resident cells and that there may be a common lymphocyte residency signature.

Since we were modeling lobar pneumonias, and prior studies revealed that immunological changes in the lung occur locally $(8,23$, 25 ), we examined the lobe specificity of the lung B cell phenotypes. The initial Sp19F exposures were restricted to the left lung lobe. EV $\mathrm{B}$ cells in the left lobes of experienced mice contained significantly greater $\mathrm{CD} 69^{+}$fractions (Figure $4 \mathrm{~B}$ ) and had significantly higher expression of CD11a (Figure 4C) compared with EV B cells of all other groups. The increase in $\mathrm{IgD}^{-} \mathrm{EV} \mathrm{B}$ cells due to experience was also restricted to the previously infected left lobe, with no such increase in the contralateral right lobes that did not have prior infections (Figure 4D). Thus, prior experience with pneumococcal infection changed only the EV B cells of the involved lobe.

The phenotype of these B cells suggested they were BRM cells. In parabiosis experiments, cells that fail to relocate to the relevant organ in the parabiont via anastomosed circulatory systems are designated as tissue-resident rather than tissue-homing cells (8). As in parabiosis experiments where the restriction of cells to the original but not partner mouse implies residency, the restriction of $\mathrm{B}$ cell phenotypes to only the ipsilateral lobe and not the contralateral lobes (Figure 4, B-D) supports the hypothesis that these cells are lung resident rather than lung homing. However, we recognized an alternative hypothesis 

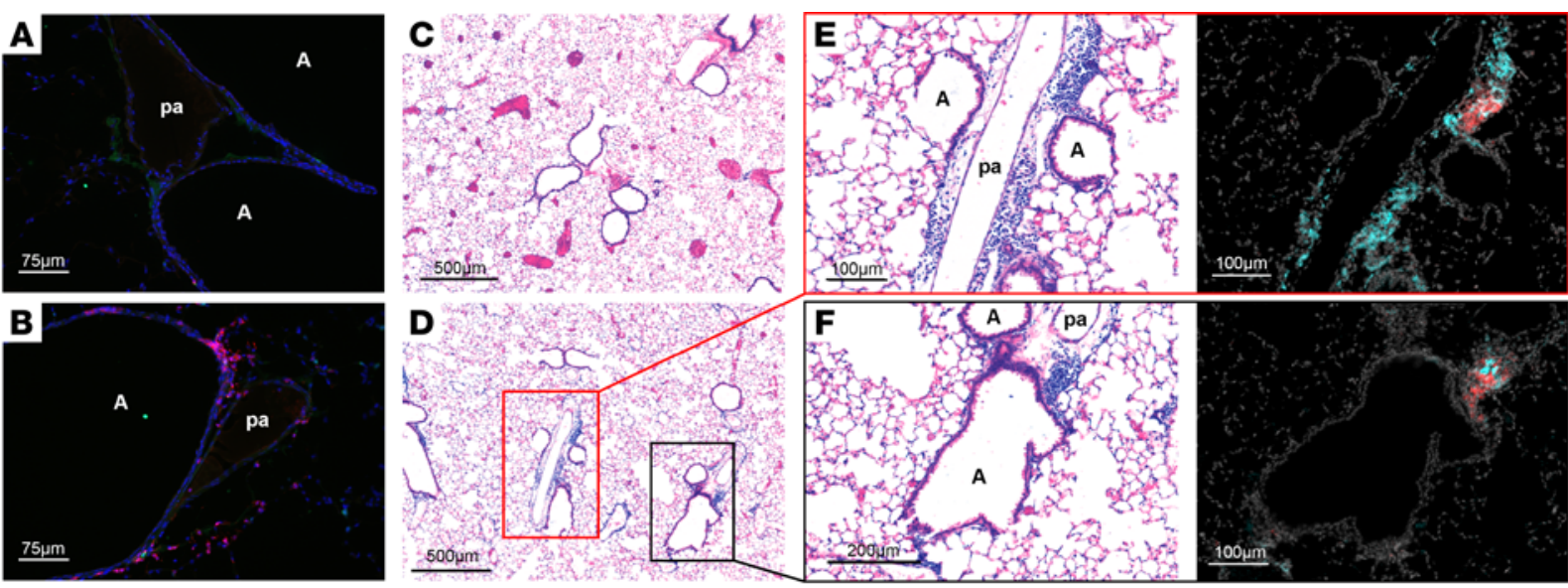

B220: RED CD4: GREEN DAPI: BLUE

CD19: RED CD4: CYAN
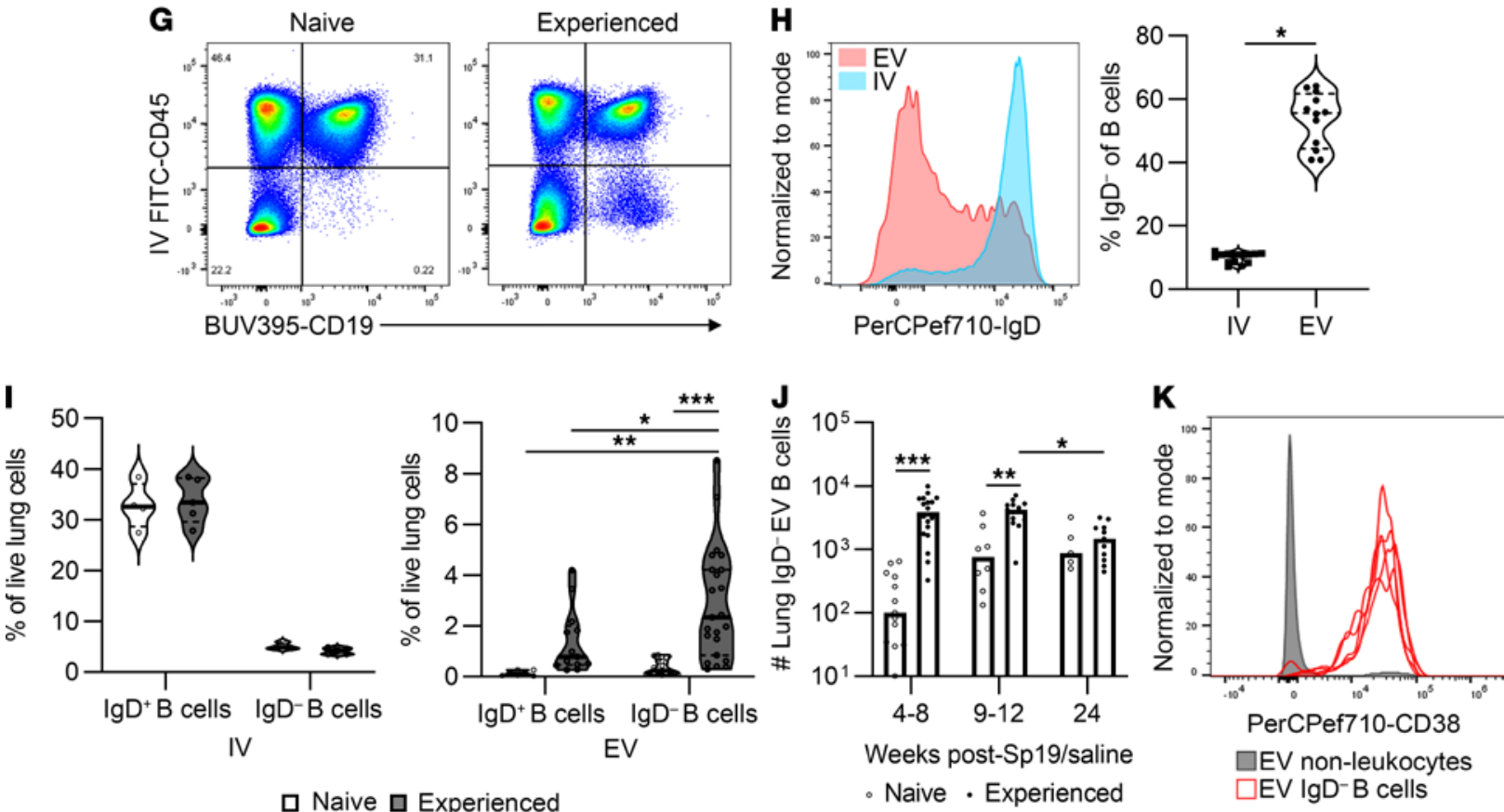

Figure 2. Pneumococcal exposure elicits clusters of EV memory B cells in the lung. Immunofluorescence staining (20x) for B220 (red), CD4 (green), and nuclei (DAPI, blue) of representative naive (A) and experienced (B) lungs. H\&E staining of representative naive (C) and experienced (D) lungs. (E and F) Left panels: Magnified view of 2 immune cell clusters in D. Right panels: Immunohistochemical staining for CD19 (red) and CD4 (cyan) in serial sections of the same lungs imaged in the left panels. Scale bars: $75 \mu \mathrm{m}$ (A and B); $500 \mu \mathrm{m}$ (C and D); $100 \mu \mathrm{m}$ (E and F). (G) Representative gating of i.v. CD45+CD19+ cells (IV B cells) and i.v. CD45-CD19+ cells (EV B cells) in lungs of naive and experienced mice. (H) IgD expression on IV and EV B cells in experienced lungs (left) and percentages of IV and EV B cells that are IgD- in experienced lungs (right; Mann-Whitney $U$ test, ${ }^{*} P<0.0001$ ). (I) Percentages of IgD+ and IgD- B cells among live lung cells in the IV and EV compartments of naive and experienced mice analyzed between 4 and 12 weeks after the previous Sp19F exposure (2-way ANOVA, ${ }^{*} P=0.029,{ }^{* *} P=0.0006,{ }^{* * *} P<0.0001$ ). (J) IgD- EV B cells in naive and experienced lungs at the indicated times after lung exposure (2-way ANOVA, ${ }^{*} P=0.01,{ }^{* *} P=0.0054,{ }^{* *} P<0.0001$ ). (K) CD38 expression on EV IgD- B cells (each red curve from 1 of 5 different mice) and on EV CD45 cells (shaded curve, representative). A, airway; pa, pulmonary artery.

may be that these B cells are not resident, but instead are circulating B cells that home only to previously infected tissue. We therefore wanted to test whether the EV lung MBCs stably remained in the lung compartment. To do so, we leveraged the fact that murine IgG2a antiCD2O antibodies deplete $\mathrm{CD} 2 \mathrm{O}^{+} \mathrm{B}$ cells in a circulation-dependent manner (30). Both IV and EV lung B cells expressed CD2O (Figure $4 \mathrm{E}, \mathrm{IgG}$-treated mice). An anti-CD2O antibody administered i.n. and i.p. reached the EV lung B cells and remained bound, as binding of a directly labeled APC-CD20 antibody was prevented in mice treated 2 weeks prior with anti-CD20 (Figure 4E). The anti-CD2O antibody effectively depleted IV B cells but had no effect on EV B cells at 4 days or 2 weeks after anti-CD2O administration (Figure 4, F and G). Because anti-CD2O bound but did not deplete the EV lung B cells, we conclude that these cells do not reenter the circulation throughout the 2-week time period examined. Thus, the lung MBCs elicited by bacterial pneumonia were lung-resident rather than lung-homing cells. 
A

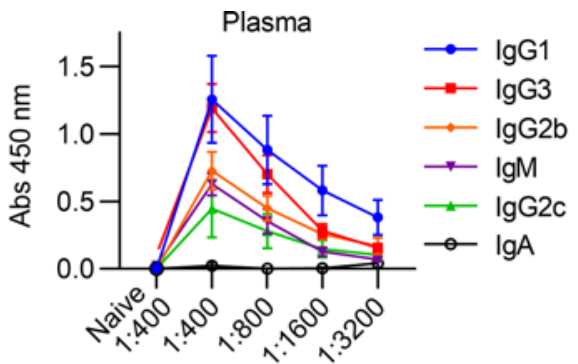

Experienced

C

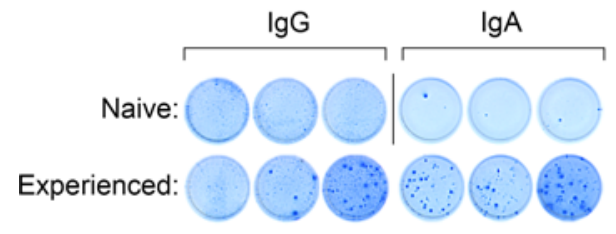

E

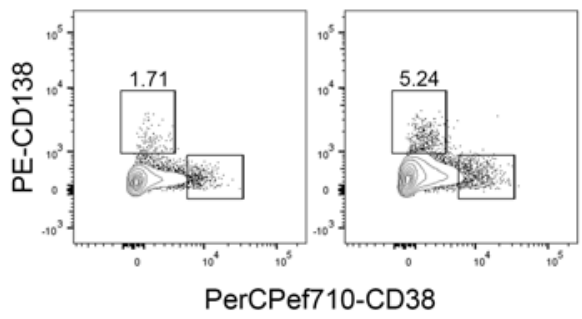

G

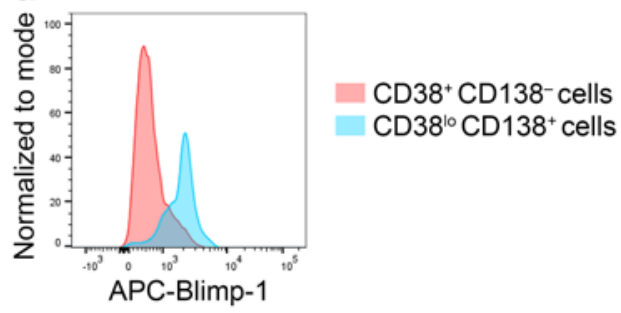

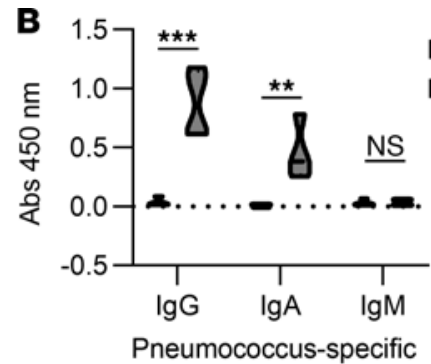
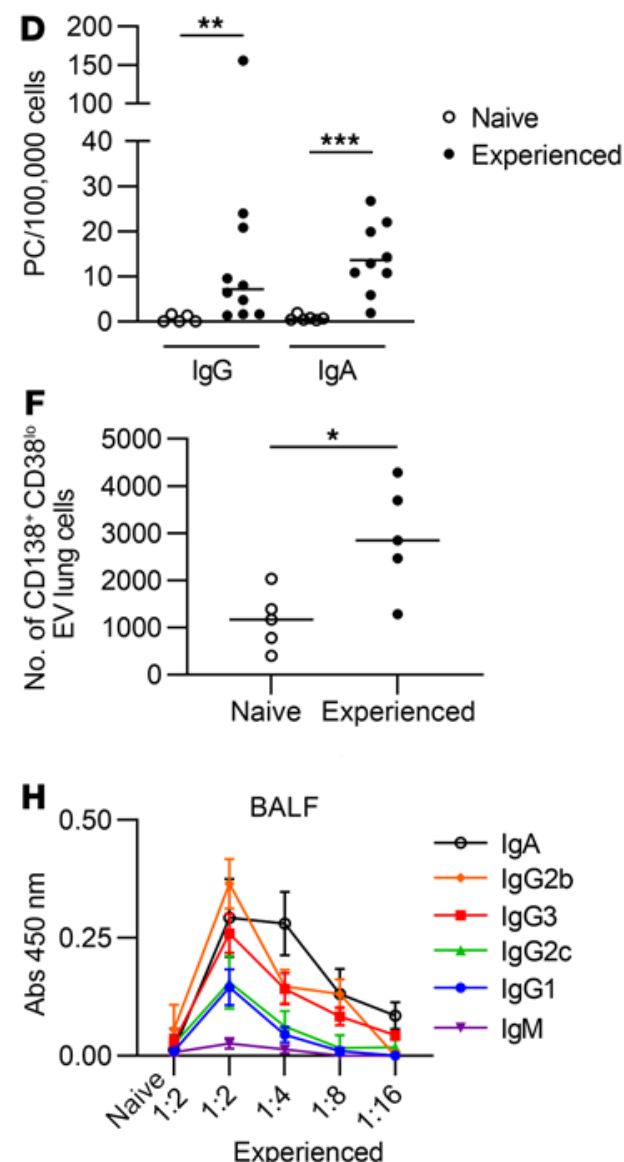

Figure 3. Pneumococcus-specific plasma cells are found in both bone marrow and lungs of experienced mice. (A) Plasma of naive and experienced mice was assessed for Sp3-reactive antibodies via ELISA. $n=3$ for naive mice and $n=6$ or more for experienced mice. (B) Pneumococcus-specific antibodies in supernatants from overnight cultures of naive or experienced bone marrow cells. $n=4$ per group. 2 -way ANOVA, ${ }^{* *} P=0.0079,{ }^{* *} P<0.0001$. Total IgG and IgA ELISpot images (C) and counts (D) of EV lung plasma cells (PC) (Mann-Whitney $U$ tests, ${ }^{* *} P=0.0037,{ }^{* *} P=0.0004$ ). Vertical line in C separates nonconsecutive wells from the same plate. Representative flow plots $(\mathbf{E})$ and quantification $(\mathbf{F})$ of EV plasma cells $\left(C D 138^{+} C D 38^{10}\right)$ and $C D 38^{+} C D 138^{-}$cells from an experienced mouse lung (Mann-Whitney $U$ test, ${ }^{*} P=0.032$ ). (C) Representative intracellular Blimp-1 expression in EV CD38 $8^{\circ} \mathrm{CD} 138^{+}$and CD38+CD138- lung cells. (H) BALF of naive and experienced mice was assessed for Sp3-reactive antibodies via ELISA. $n=4$ for naive mice and $n=7$ or more for experienced mice.

Human lung BRM cells. Since pneumococcus and influenza are both common causes of lung infection in humans (17) and both lead to deposition of lung BRM cells in mice (Figures 2 and 4; and ref. 8), we hypothesized that human lungs may contain BRM cells. Disease-free donor samples from lung tissue wedge resections or lobectomies were assessed via flow cytometry (Figure $5 \mathrm{~A}$ ). Consistent with a prior report (2), significantly more $\mathrm{CD} 4^{+} \mathrm{T}$ cells than $\mathrm{B}$ cells were observed in the human lungs (Figure 5B). Of the $\mathrm{CD} 19^{+}$cells in these lungs, the majority $(\sim 65 \%)$ were positive for the human MBC marker CD27, and the remaining cells were $\operatorname{IgD}^{+}$(Figure $5 \mathrm{C}$ ). Most of the MBCs $\left(\mathrm{CD} 19^{+} \mathrm{CD} 27^{+}\right)$as well as the $\mathrm{CD} 4^{+} \mathrm{T}$ cells in these lung samples were
$\mathrm{CD}^{2} 9^{+}$, whereas most naive $\mathrm{B}$ cells $\left(\mathrm{CD} 19^{+} \mathrm{IgD}^{+}\right)$in the same lungs were not (Figure 5D). The percentages of lung $\mathrm{CD} 4^{+} \mathrm{T}$ cells that were $\mathrm{CD}^{2} 9^{+}$in our samples matched well to lung $\mathrm{T}$ cell data from studies of humans with no known lung disease $(2,31)$, suggesting our samples appropriately represented human lung immunology. The majority of MBCs in these lungs were class switched, with only approximately $17 \%$ expressing IgM (Figure 5E). The CD69 ${ }^{+} \mathrm{MBC}$ s were negative for CD38 (Figure 5F), indicating they were not germinal center or plasma cells (32). In addition to being a residence marker, CD69 is expressed by activated $B$ cells, but almost all the human CD $69^{+} \mathrm{MBC}$ s were negative for CD83 (Figure 5F), another marker of B cell activation (33). 
A
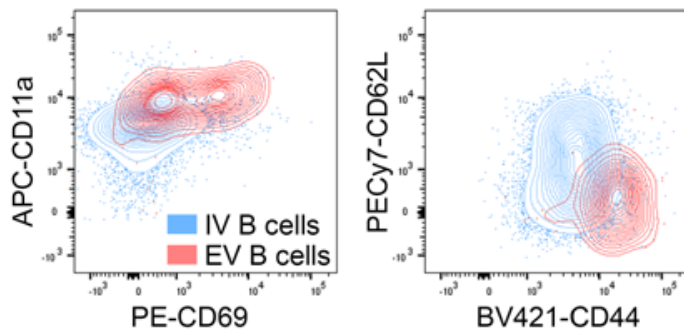

BV421-CD44
B

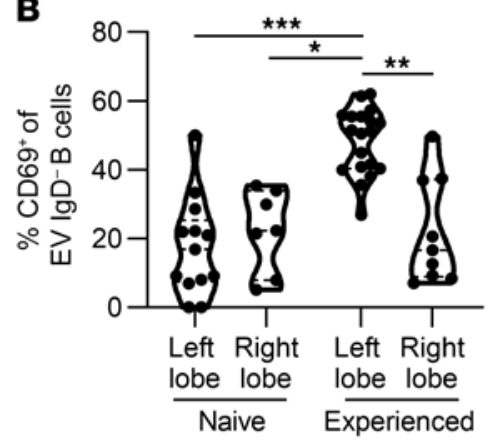

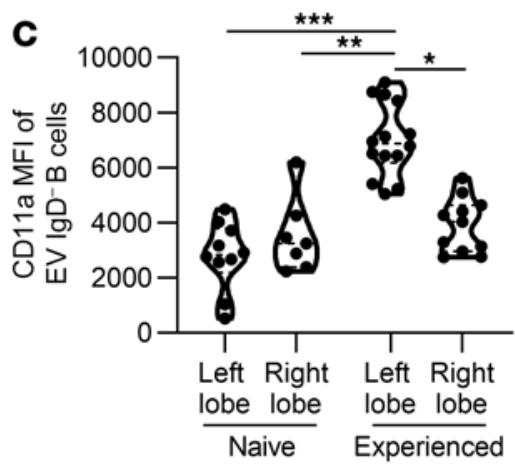
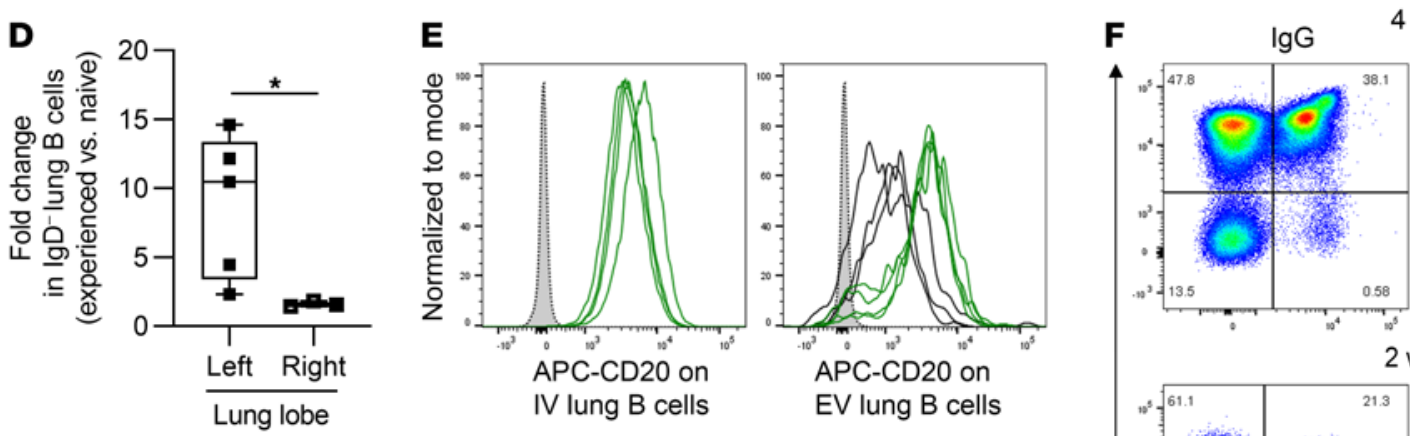

4 days

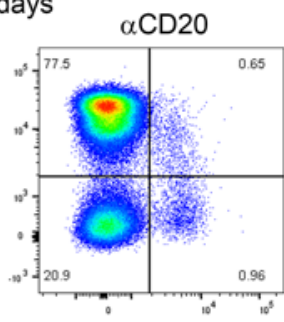

2 weeks

CD20 FMO $\square \alpha$ CD20 treated $\square$ IgG treated
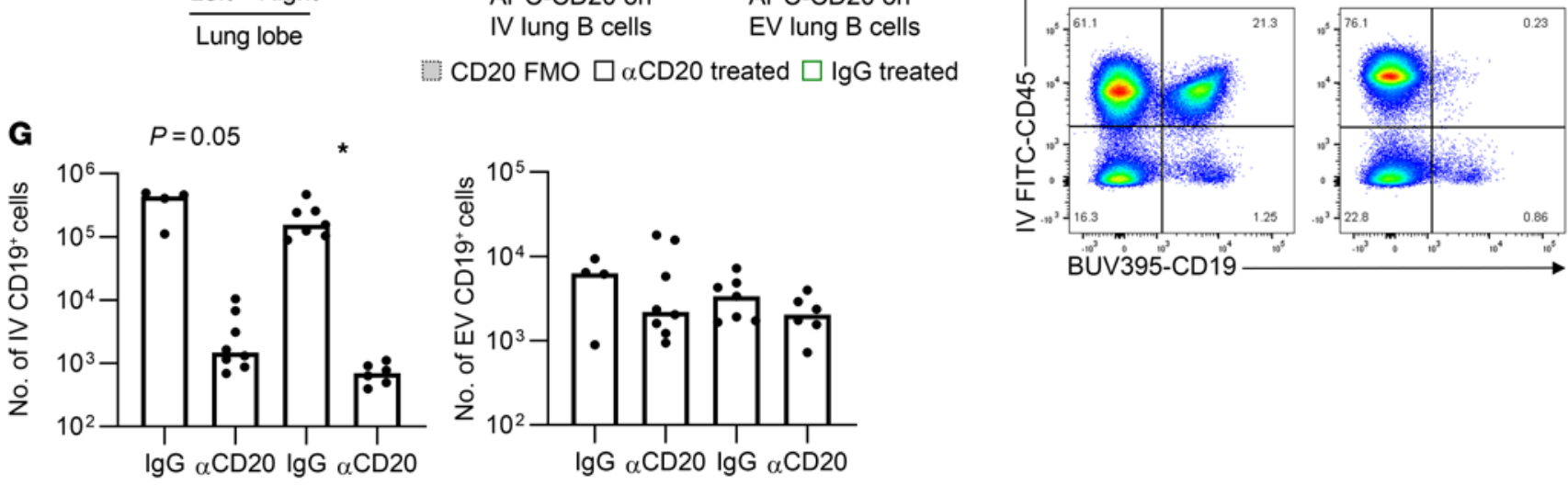

Figure 4. Lung B cells in experienced mice are resident. (A) Representative flow cytometry plots showing expression of CD69, CD11a, CD62L, and CD44 on IV and

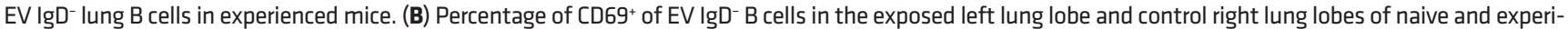
enced mice (1-way ANOVA, ${ }^{*} P=0.0054,{ }^{* *} P=0.0021$, $\left.{ }^{* *} P<0.0001\right)$. (C) Median fluorescence intensity (MFI) of CD11a on EV IgD- B cells in the exposed left lung lobe and control right lung lobes of naive and experienced mice (1-way ANOVA, ${ }^{*} P=0.0047,{ }^{* *} P=0.0017,{ }^{* *} P<0.0001$ ). (D) Fold change in EV lgD' lung B cells between saline-treated and experienced mice in the exposed left lung lobe and unexposed control right lobe. Each dot represents the average fold change in 1 experiment including at least 3 naive and 3 experienced mice (Mann-Whitney $U$ test, ${ }^{*} P=0.036$ ). (E) Binding of a fluorescent anti-CD2O antibody to lung $B$ cells from mice treated 2 weeks previously with isotype control IgG or anti-CD20 (5D2 clone). FMO, fluorescence minus one. Representative flow cytometry plots (F) and quantification CD19+ cells (G) in lung compartments 4 days or 2 weeks after anti-CD20 or lgG treatment of experienced mice (Kruskal-Wallis test, ${ }^{*} P=0.002$ ).

Therefore, it is likely that CD69 is a marker of resident, as opposed to recently activated, cells in this context. These data provide evidence that human lungs contain class-switched BRM cells.

Antibody secretion from lung BRM cells. After observing that pneumococcal exposures elicit lung BRM cells, we wanted to more deeply explore the phenotypic and functional aspects of these cells in mice. Although the EV lung B cell compartment included naive B cells $\left(\operatorname{IgD}^{+} \operatorname{IgM}{ }^{+}\right)$, a larger fraction was $\operatorname{IgM}^{+} \operatorname{IgD}^{-}$than in the spleen or IV compartment from the lung (Figure 6A). Additionally, an appreciable proportion of class-switched B cells was observed in the lung EV tissue - in contrast to the spleen and IV lung cell populations - highlighting the enrichment of switched memory cells at this site (Figure 6A). Of the class-switched EV lung B cells, the majority were of an IgG isotype, with a small fraction of $\operatorname{IgA}^{+} \mathrm{B}$ cells (Figure $6 \mathrm{~B}$ ).
In mice, the markers PD-L2, CD73, and CD80 distinguish functional MBC phenotypes (34). In lung B cells of experienced mice, the expression of all 3 markers was restricted, as expected, to $\mathrm{IgD}^{-}$ cells, and the EV compartment was heavily enriched with B cells bearing these markers compared with IV B cells in the same mice (Figure 6C). Importantly, the EV B cells in experienced lungs predominantly expressed more than one of these markers (Figure 6, D and E). Coexpression of at least 2 of these 3 memory markers distinguishes MBCs likely to differentiate into antibody-secreting cells (ASCs) upon reactivation (34).

To test whether the lungs of experienced mice contained MBCs that could be reactivated into ASCs, we stimulated total splenic $\mathrm{B}$ cells and EV lung B cells from naive or experienced mice. After 4 days of ex vivo culture followed by IgG ELISpot analysis, spots 
A

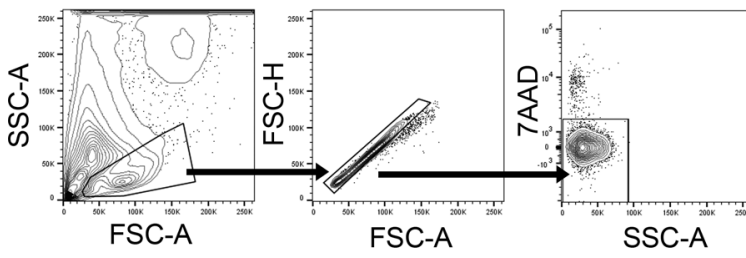

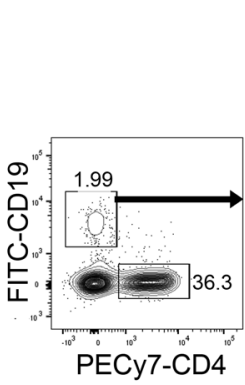

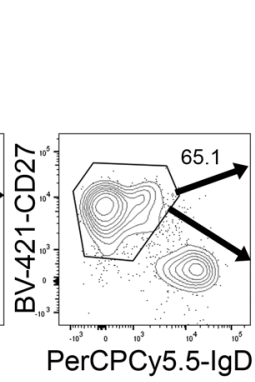

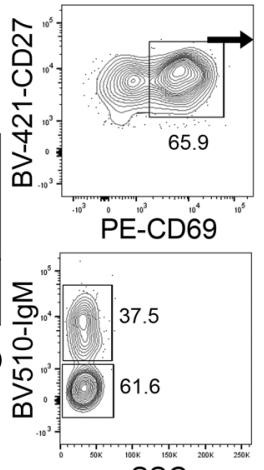

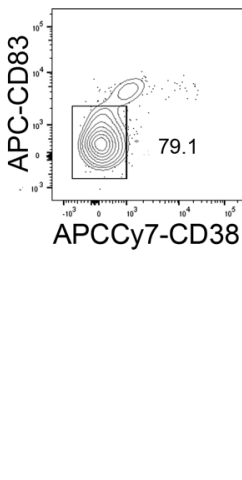

SSC

B

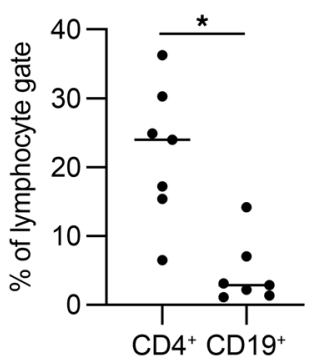

c

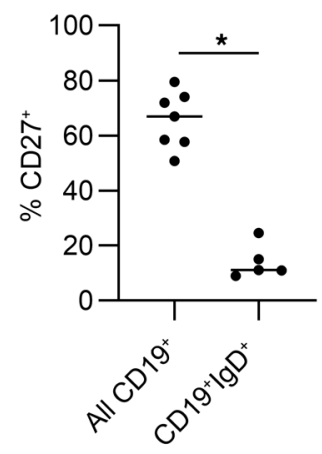

D

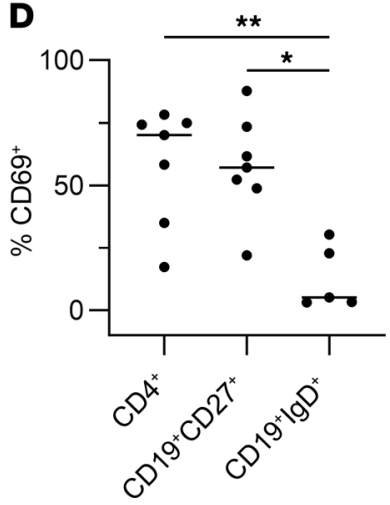

E

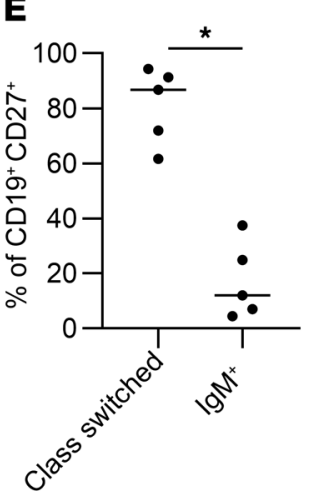

$\mathbf{F}$

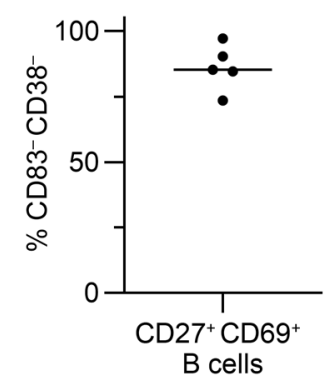

Figure 5. Human lungs are enriched for B cells bearing a resident memory phenotype. (A) Normal tissue from wedge resection or biopsy samples from humans with lung cancer were collagenase digested and analyzed via flow cytometry using the gating scheme shown. (B-F) Various cell surface phenotypes of $B$ and T cells in the human lung digests. (B) Percentage of $C D 4^{+}$and $C D 19^{+}$cells among live, single cells (Mann-Whitney $U$ test, ${ }^{*} P=0.0023$ ). (C) Percentage of $C D 27^{+}$cells among all CD19+ cells and among naive (CD19+IgD+) B cells (Mann-Whitney $U$ test, $\left.{ }^{*} P=0.0025\right)$. (D) Percentage of CD69+ cells among CD4 ${ }^{+}$ cells, memory $B$ cells $\left(C D 19+C D 27^{+}\right)$, and naive B cells (Kruskal-Wallis test, $\left.{ }^{*} P=0.021,{ }^{*} P=0.049\right)$. (E) Percentage of memory B cells that are class switched (Class sw.) and percentage that are IgM+ (Mann-Whitney $U$ test, $\left.{ }^{*} P=0.0079\right)$. (F) Percentage of resident memory $B$ cells $\left(C D 27^{+} C D 69{ }^{+} C D 19+\right)$ negative for the $B$ cell activation marker CD83 and for CD38.

representing reactivated $\mathrm{MBCs}$ were detected more frequently in wells containing stimulated cells from experienced mouse lungs than in wells with unstimulated cells from experienced mice or stimulated cells from naive mice (Figure 6, F and G). Although stimulation also elicited ASC differentiation in spleen cells, there was not a significant difference in the number of spleen IgG ASCs between naive and experienced mice (Figure 6G). Supernatants from ex vivo cultures of stimulated experienced, but not stimulated naive, mouse lung cells contained IgG and IgM antibodies that bound to an acapsular strain of Sp3, indicating they were specific to serotype-independent pneumococcal antigens. Again, stimulated spleen cells from both naive and experienced mice were able to produce pneumococcus-reactive IgG and IgM, with no significant differences between the groups (Figure 6H). These results show that the spleens of naive mice contained B cells that could be stimulated to secrete antibody against pneumococcal antigens, and there was no significant enrichment of such B cells after lung exposure to pneumococcus. These splenic B cells likely consist of innate-like marginal zone or B 1 B cells surviving culture only with stimulation and able to produce pneumococcus-binding antibodies (35). In the lung, however, prior pneumococcal exposure is required in order to generate pneumococcus-specific MBCs. To see whether local antibody secretion in the lung occurred following pneumococcal challenge in vivo, we analyzed the BALF of experienced mice at baseline and 96 hours after Sp3 challenge. The BALF of experienced mice after challenge contained significantly more IgG, IgA, and IgM antibodies able to bind an acapsular Sp3 strain (Figure 6I). These findings may indicate that BRM cells in the experienced lung are reactivated by a heterotypic pneumococcal challenge to locally secrete serotype cross-reactive antibodies. Thus, the lungs of mice previously exposed to pneumococcus are enriched with pneumococcus-specific lung BRM cells that are poised to rapidly secrete antibody upon stimulation.

Contributions of systemic B cell immunity to lung protection. That mice recovered from pneumococcal infections are later protected from a serotype-mismatched challenge with Sp3 (Figure 1 and ref. 23) spurred our investigation of whether B cell-related immune mechanisms contribute to this protection. We found that this protection is partially dependent on B cell-mediated immunity, as evidenced by the increased bacterial burdens (Figure 7A) and morbidity (Figure 7B) in experienced B cell-deficient $\left(I g h m^{\text {tmlcgn }} ; \mu \mathrm{MT}\right)$ mice compared with experienced C57BL/6 (B6) mice after Sp3 infection. Notably, although $\mu \mathrm{MT}$ mice in our model develop a slightly diminished lung TRM cell pool compared with B6 mice (Supplemental Figure $4, \mathrm{~A}$ and $\mathrm{B}$ ), experienced $\mu \mathrm{MT}$ mice still generate as much lung $\mathrm{CD}^{+}{ }^{+} \mathrm{T}$ cell-derived IL-17A after $\mathrm{Sp} 3$ challenge as experienced B6 mice (Supplemental Figure 4, C and D; and ref. 23). Therefore, 
A
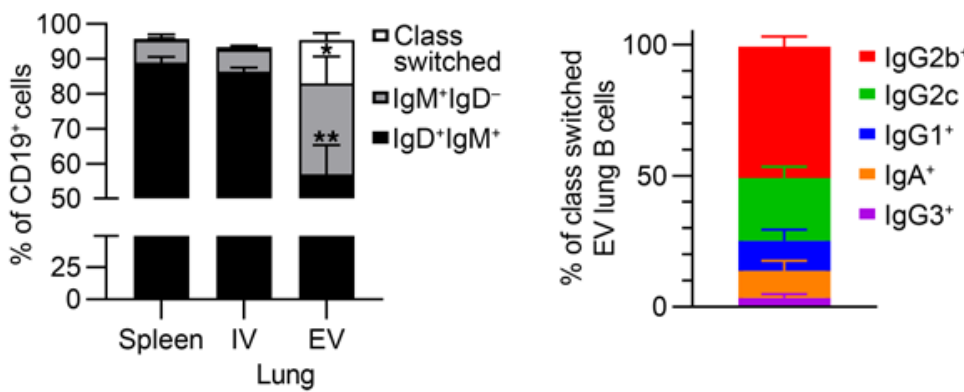

D

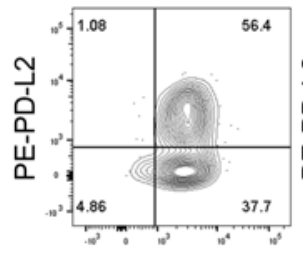

BV421-CD80
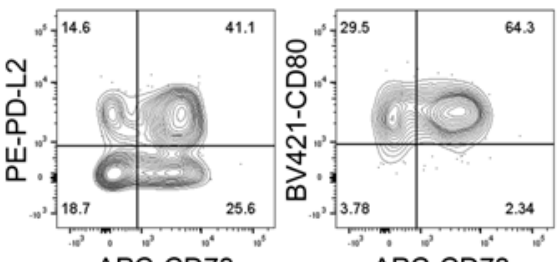

$\mathbf{F}$

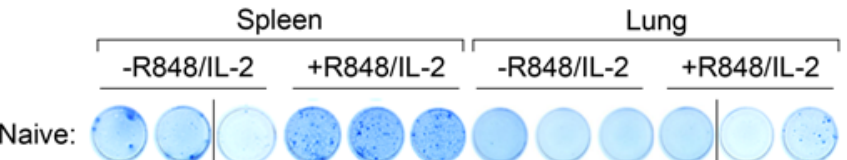

Experienced:
B

APC-CD73
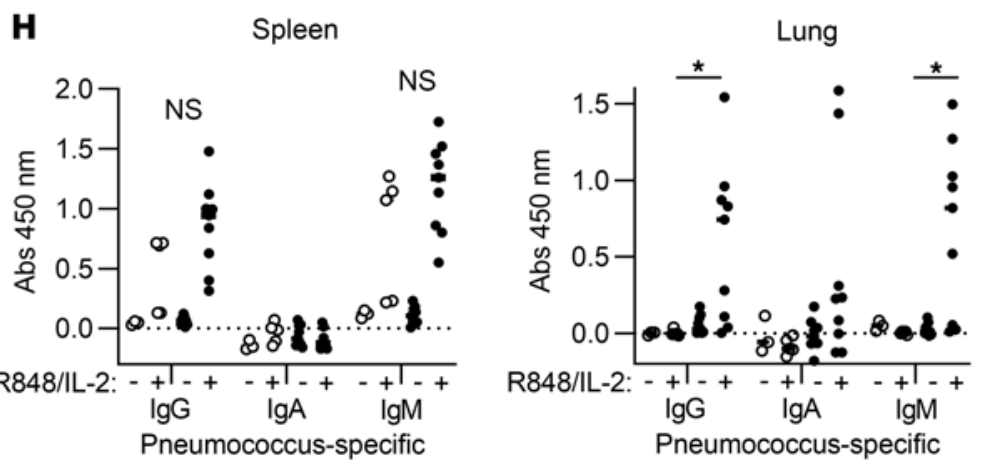
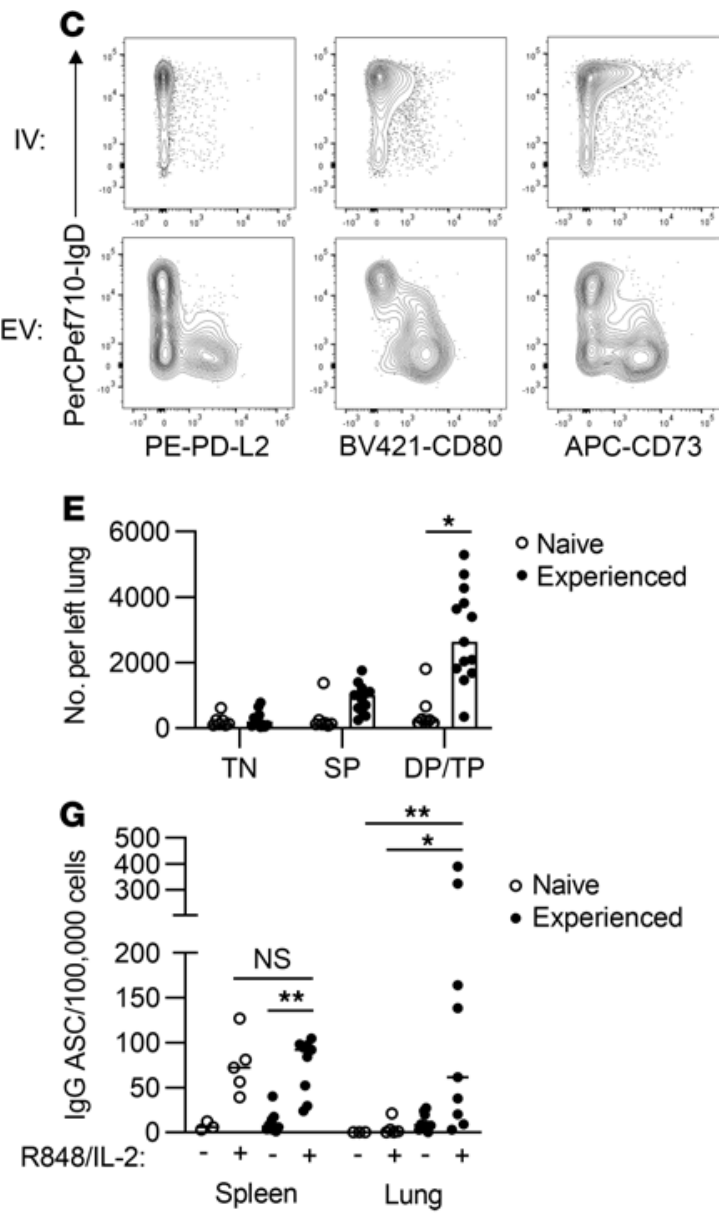

I

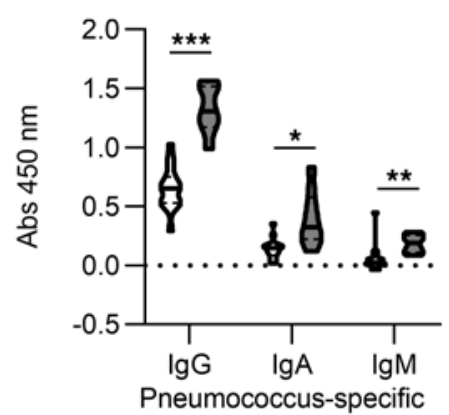

- Experienced

$0 \mathrm{hr}$

$96 \mathrm{hrs} \mathrm{Sp3}$

- Naive • Experienced

Figure 6. Lung BRM cells are poised to secrete antibody. (A) Distribution of naive, IgM+IgD-, and class-switched B cells in experienced lungs and spleens (2-way ANOVA comparing isotypes across compartments, $n=3$ for spleen, 5 for lung; ${ }^{*} P=0.0022$ vs. spleen, $P=0.0009$ vs. IV lung; ${ }^{*} P<0.0001$ vs. either compartment). (B) Experienced EV lung IgM-IgD- B cell isotypes. $n=5$. Representative flow plots from experienced lungs showing MBC marker expression on IgD- EV B cells (C) and coexpression (D) as quantified in $\mathbf{E}$, where numbers of triple-negative (TN), single-positive (SP), and double- or triple-positive (DP/TP) B cells are shown (2-way ANOVA comparing each marker category between naive and experienced mice, $\left.{ }^{*} P<0.0001\right)$. Total IgC ELISpot image (F) and counts (G) of spleen and EV lung B cells after ex vivo culture (Kruskal-Wallis test for each organ, ${ }^{*} P=0.028,{ }^{*} P=0.0035$ for spleen, $P=0.006$ for lung). Vertical lines in $\mathbf{F}$ separate images within a group obtained from different plates. (H) Pneumococcus-specific antibody levels in ex vivo culture supernatants from $\mathbf{F}$ and $\mathbf{G}$ (Kruskal-Wallis test for each isotype within each organ, ${ }^{*} P=0.0039$ for IgG, $P=0.0064$ for IgM). (I) Pneumococcus-specific antibody in experienced BALF at baseline or after Sp3 infection (Mann-Whitney $U$ test for each isotype, $n=15$ for baseline, $n=8$ for 96 hours; ${ }^{*} P=0.0018,{ }^{* *} P=0.0007,{ }^{* *} P<0.0001$ ).

the less-robust protection of experienced $\mu \mathrm{MT}$ mice was not due to a failure to generate effective lung $\mathrm{T}$ cell memory, as has been observed in other infection settings (36).

The most likely contribution of B cells to antipneumococcal defense is via antibodies. To determine whether heterotypic antibodies could be sufficient to confer protection against pneumococcus in the lung, we infected the lungs of naive mice with $\mathrm{Sp} 3$ that was pre-opsonized by plasma collected from either naive mice or mice recovered from Sp19F infections (without or with heterotypic antibodies, respectively; Figure 3A). This plasma from experienced 
A

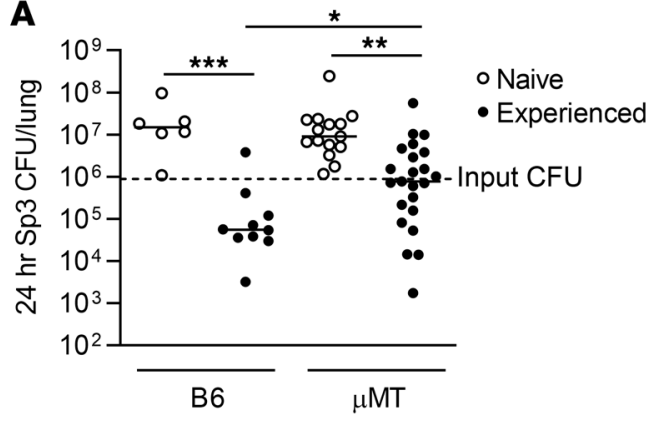

C

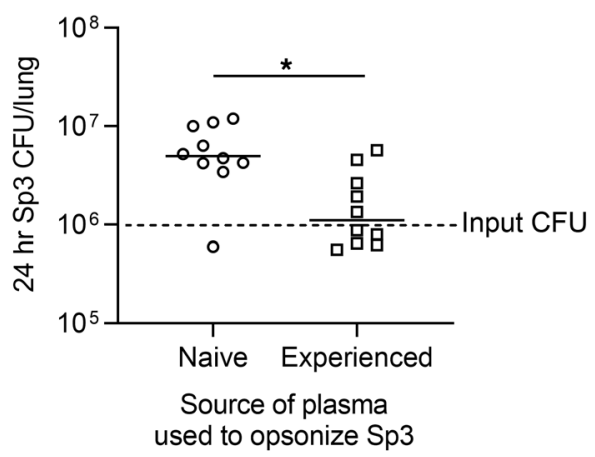

E

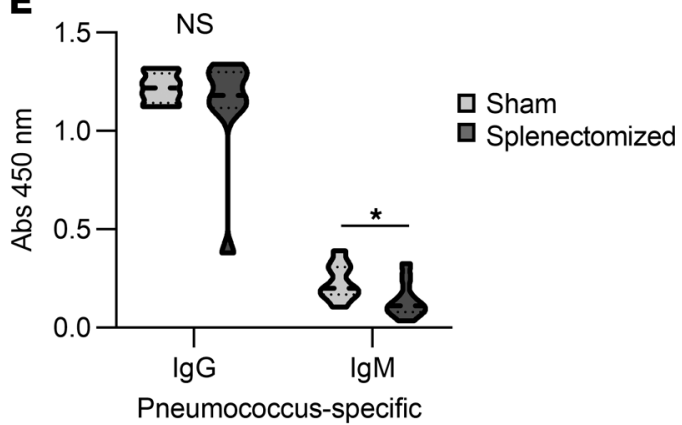

B

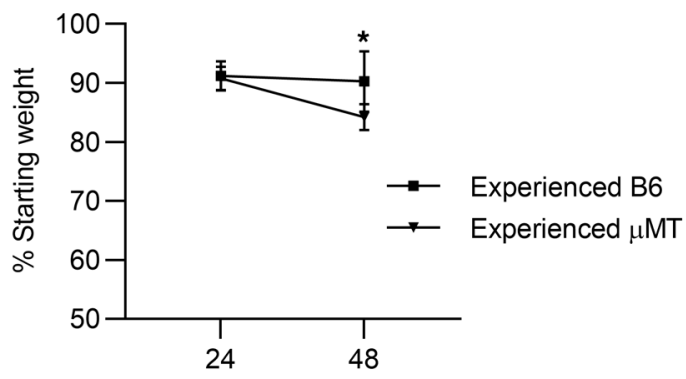

D

Hours post-Sp3

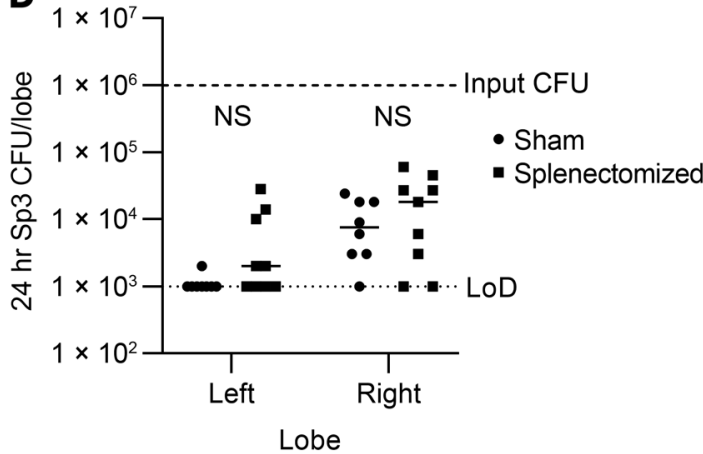

$\mathbf{F}$

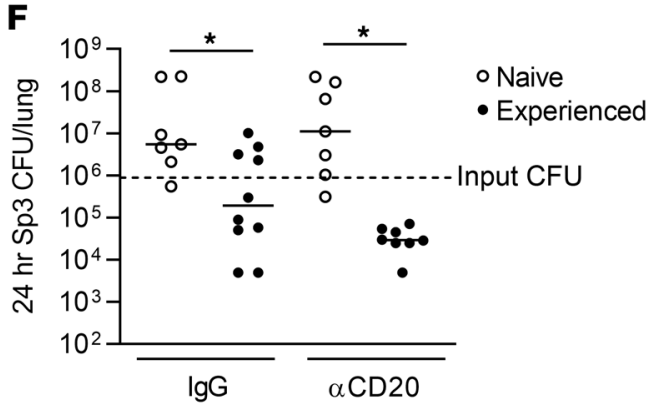

Figure 7. Systemic B cell immunity may contribute to, but is not required for, serotype-independent lung antipneumococcal immunity in experienced mice. (A) Twenty-four-hour lung Sp3 burdens in naive and experienced B6 or $\mu$ MT mice (2-way ANOVA, ${ }^{*} P=0.013,{ }^{* *} P=0.0004,{ }^{* *} P<0.0001$ ). (B) Weight loss after Sp3 challenge in experienced B6 and $\mu \mathrm{MT}$ mice (2-way ANOVA comparing mouse strains within each time point, $n=16 \mathrm{~B} 6$ and $n=9 \mu \mathrm{MT} ;{ }^{*} P=0.0004$ ). (C) Plasma of experienced or naive mice was used to pre-opsonize Sp3 prior to bacterial instillation in naive mice and determination of 24-hour lung CFU (Mann-Whitney $U$ test, ${ }^{*} P=0.01$ ). Twenty-four-hour lung Sp3 CFU (D; no significant differences by 2-way ANOVA) and plasma antipneumococcal antibody titers $(\mathbf{E})$ were determined in experienced mice that were splenectomized or given sham surgery 3 weeks prior to $\mathrm{Sp} 3$ infection (for $\mathbf{E}$, Mann-Whitney $U$ test for each isotype, $\left.{ }^{*} P=0.046\right)$. (F) Twenty-four-hour lung Sp3 burdens in naive and experienced mice treated 4 days prior with isotype control or anti-CD20 (2-way ANOVA, ${ }^{*} P=0.0024$ for IgG, $P=0.0027$ for anti-CD20). LoD, limit of detection.

mice conferred protection compared with plasma from naive mice (Figure 7C), supporting the hypothesis that heterotypic antibodies can contribute to lung defense against pneumococcus.

The spleen contains B 1 and marginal zone B cells that can produce heterotypic antibodies and contribute to antipneumococcal immunity (refs. 35, 37) (Figure 6H). To test whether these cells contributed to serotype-independent antipneumococcal lung defense, we compared Sp3 bacterial burdens in experienced mice that were splenectomized 3 weeks prior to Sp3 challenge and experienced control mice given sham surgeries. All experienced mice demonstrated effective defense against intrapulmonary Sp3, with no significant differences in lung bacteria between the splenectomized and control groups (Figure 7D). Splenectomy reduced pneumococcus-specific IgM in the blood of experienced mice, but not antipneumococcal IgG (Figure 7E). Thus, B cells in the spleen contributed to pneumococcus-specific IgM in the circulation of experienced mice, but were dispensable for serotype-independent lung immunity against local pneumococcal infection.

Since B cells - but not those originating in the spleen - were essential to local protection (Figure 7, A and D), we considered whether circulating B cells migrating through blood and lymph nodes may be required for the lung defense of experienced mice. Knowing that the anti-CD2O antibody effectively depletes circulating B cells while leaving lung BRM cells intact (Figure 4), we tested whether anti-CD20 treatment affected lung defense in experienced mice. Depletion of all circulating B cells in experienced B6 mice via 4 days of anti-CD20 treatment had no effect on 24-hour clearance of Sp3 (Figure 7F). These data suggest that B cells, but not splenic or 
A
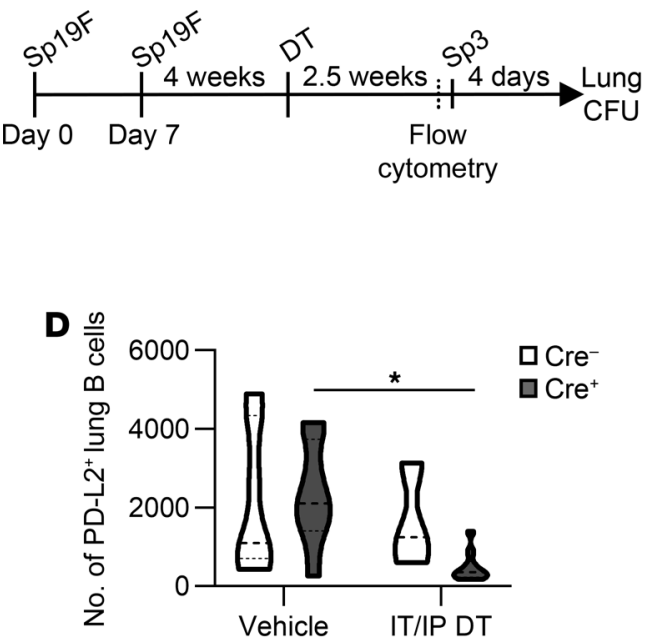

G

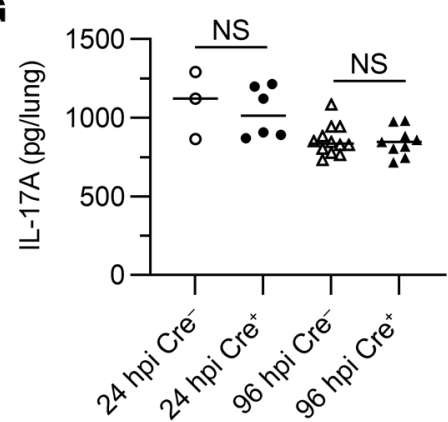

J

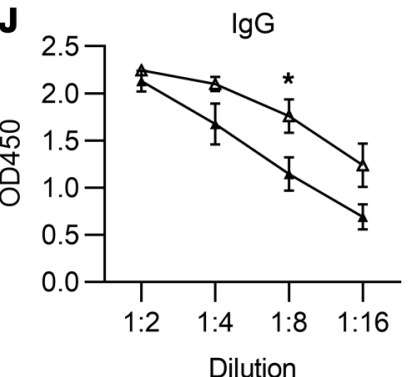

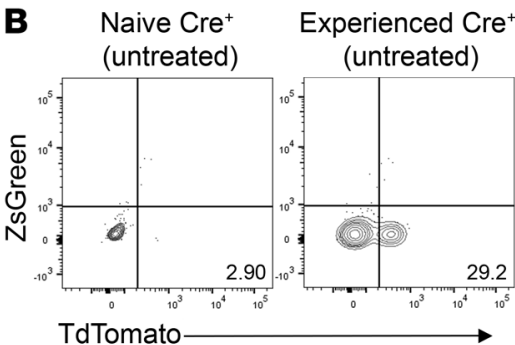

C Experienced $\mathrm{Cre}^{+}$Experienced $\mathrm{Cre}^{+}$
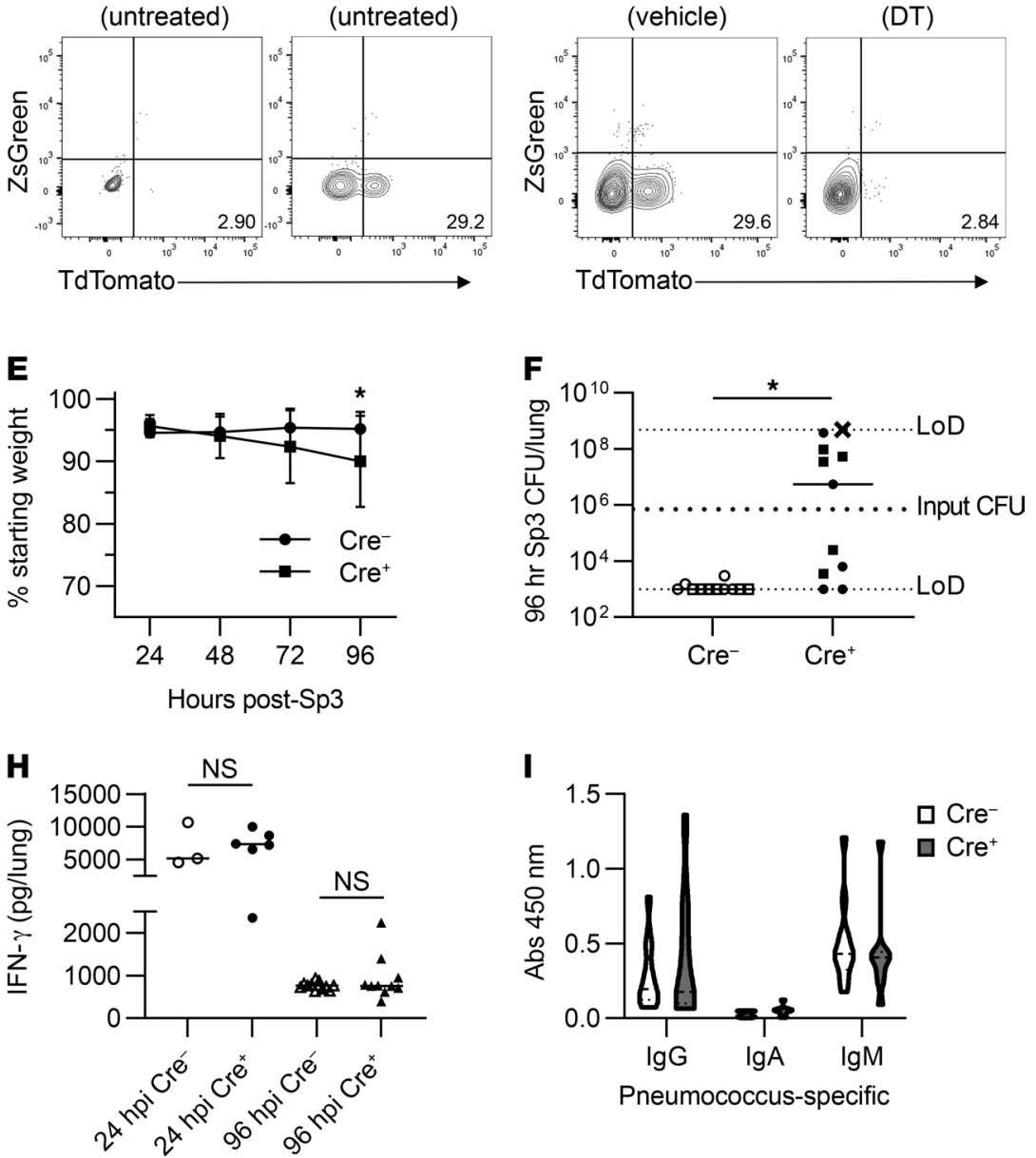
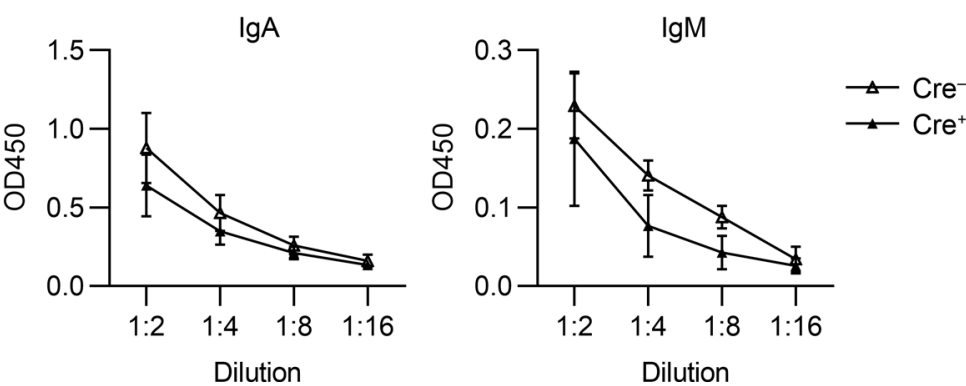

Figure 8. Lung PD-L2+ MBC are required for optimal serotype-independent antipneumococcal lung immunity and local IgC production. (A) Timeline of treatments in PZTD mice. Representative plots of EV lung B cells in naive and experienced Cre ${ }^{+}$mice (B) and in experienced Cre ${ }^{+}$mice with or without DT (C). (D) Quantification of PD-L2+ EV B cells in lungs of experienced Cre- and Cre ${ }^{+}$mice with or without DT (2-way ANOVA, $n=7$ for vehicle-treated Cre-, $n=3$ for DT-treated $\mathrm{Cre}^{-}, n=8$ for vehicle-treated $\mathrm{Cre}^{+}$, and 10 for DT-treated Cre ${ }^{+}$mice; $\left.{ }^{*} P=0.029\right)$. (E) Weight loss after Sp3 challenge in DT-treated experienced Cre ${ }^{-}$and $\mathrm{Cre}^{+}$mice (2-way ANOVA comparing genotypes at each time point, $n=11$ per genotype; ${ }^{*} P=0.0093$ ). (F) Ninety-six-hour lung Sp3 burdens in DT-treated experienced $\mathrm{Cre}^{-}$and $\mathrm{Cre}^{+}$mice (Mann-Whitney $U$ test, ${ }^{*} P=0.0004$ ). Squares, female; circles, male for Cre ${ }^{+}$group. One male mouse (identified with an X) died shortly before lung harvest. IL-17 (G) and IFN- $\gamma(\mathbf{H})$ levels in lung homogenates of DT-treated experienced PZTD mice after 24 or 96 hours of Sp3 infection (no significant differences by Mann-Whitney $U$ tests within each time point). (I) Plasma of mice from F was assessed for Sp3-reactive antibodies via ELISA (no significant differences by Mann-Whitney $U$ test within each isotype). (J) BALF from experienced DT-treated Cre- and Cre+ PZTD mice was collected in a single experiment 96 hours after i.n. Sp3 infection and assessed for Sp3-reactive antibodies via ELISA. Two-way ANOVAs, $n=6$ for Cre- mice and $n=4$ for $\mathrm{Cre}^{+}$mice; ${ }^{*} P=0.048$. hpi, hours after infection.

circulating B cells, contribute to lung defense in experienced mice, implicating functional roles for B cells in other sites.

Pneumonia protection by lung BRM cells. We hypothesized that lung BRM cells may be contributing to bacterial clearance, but there are no means of selectively deleting these cells. Since more than half of the $\mathrm{IgD}^{-}$lung EV B cells were PD-L2+ (Figure 6, C and D), we devised a strategy to deplete PD-L2 ${ }^{+} \mathrm{B}$ cells specifically. When crossing CD19-Cre mice are crossed into the PD-L2-ZsGreen-TdTomato-diphtheria toxin knockin and inducible knockout (PZTD) background (38), all PD-L2 ${ }^{+}$and only $\mathrm{PD}-\mathrm{L} 2^{+}$cells are fluorescent, 
with CD19-PD-L2 ${ }^{+}$cells in both $\mathrm{Cre}^{-}$and $\mathrm{Cre}^{+}$mice expressing the fluorescent ZsGreen protein; while CD19 ${ }^{+} \mathrm{PD}-\mathrm{L} 2^{+}$cells in $\mathrm{Cre}^{+}$mice express the fluorescent TdTomato protein as well as the diphtheria toxin (DT) receptor. Resolution of Sp19F infections in these mice (Figure 8A) elicited PD-L2+ (TdTomato ${ }^{+}$) EV lung B cells (Figure 8B; gating, Supplemental Figure 5A). Treating experienced $\mathrm{Cre}^{+}$mice with DT eliminated this PD-L2 ${ }^{+} \mathrm{B}$ cell population from the lungs (Figure $8, \mathrm{C}$ and D). In contrast, DT treatment of experienced $\mathrm{Cre}^{-}$mice did not significantly impact lung EV PD-L2 ${ }^{+}$B cells (Figure 8D). DT also decreased pleural PD-L2 ${ }^{+}$B cells (which are primarily B 1 B cells) in $\mathrm{Cre}^{+}$mice (Supplemental Figure 5, B and C). However, pleural fluid $\mathrm{CD} 19^{+} \mathrm{B}$ cells do not change in frequency in response to prior pneumococcal experience (Supplemental Figure 5, D and E), nor did it appear that pleural B 1 cells infiltrated the lungs of mice challenged with Sp3 (Supplemental Figure 5, F and G), so these cells are unlikely to mediate improved immunity in experienced mice. To determine roles of PD-L2 ${ }^{+} \mathrm{B}$ cells in experienced mice, we allowed resolution of Sp19F infections in $\mathrm{Cre}^{+}$and $\mathrm{Cre}^{-}$mice, after which DT was delivered to all mice to deplete lung PD-L2 ${ }^{+} \mathrm{BRM}$ cells from $\mathrm{Cre}^{+}$mice. Two and a half weeks later, all mice were challenged with Sp3 (Figure 8A). The $\mathrm{Cre}^{+}$mice exhibited significantly more morbidity as assessed by weight loss after 4 days of Sp3 infection (Figure 8E). This morbidity was likely driven by the increased bacterial burdens in the lungs of $\mathrm{Cre}^{+}$mice, since in the majority of $\mathrm{Cre}^{-}$but not $\mathrm{Cre}^{+}$mice, bacteria were reduced to below the limit of detection over the 4 days of infection (Figure $8 \mathrm{~F}$ ). Because $\mathrm{CD} 4^{+} \mathrm{T}$ cells and their cytokines contribute to lung defense $(23,25)$ and can be stimulated by antigen presentation from B cells (39), we tested whether PD-L2 ${ }^{+}$B cells were needed for this arm of pulmonary immunity by comparing the levels of $\mathrm{T}$ cell cytokines in DT-treated experienced $\mathrm{Cre}^{-}$and $\mathrm{Cre}^{+}$mice following 24 or 96 hours of Sp3 infection. No significant differences in either lung IL-17A (Figure 8G) or lung IFN- $\gamma$ (Figure 8H) were observed between genotypes, implying that lung $\mathrm{PD}-\mathrm{L}_{2}{ }^{+} \mathrm{B}$ cells are not required for lung $\mathrm{T}$ cell responses following pneumococcal reinfection. This finding is consistent with the establishment of a functional lung TRM cell pool in $\mu \mathrm{MT}$ mice (Supplemental Figure 4). Circulating heterotypic antipneumococcal antibody levels also did not differ between DT-treated experienced $\mathrm{Cre}^{-}$and $\mathrm{Cre}^{+}$mice (Figure 8I), indicating that PD-L2 ${ }^{+}$lung B cells did not substantially contribute to circulating antibody pools. Because pneumococcus-reactive antibody levels, particularly $\operatorname{IgG}$, increased within the air spaces of the lung during infection (Figure 6I), we hypothesized that local lung antibodies may require $\mathrm{PD}-\mathrm{L} 2^{+}$lung $\mathrm{B}$ cells. To test whether depletion of the PD-L2 ${ }^{+}$ lung B cells led to a decrease in airspace antibodies, we collected the BALF of experienced DT-treated $\mathrm{Cre}^{-}$and $\mathrm{Cre}^{+}$PZTD mice 96 hours after Sp3 infection and looked for antibodies able to bind an acapsular pneumococcal strain. Levels of pneumococcus-specific BALF IgG were significantly lower in $\mathrm{Cre}^{+}$compared with $\mathrm{Cre}^{-}$mice (Figure 8J). The pneumococcus-specific IgG in the air spaces of $\mathrm{Cre}^{+}$mice lacking PD-L2 ${ }^{+} \mathrm{B}$ cells was not eliminated but was less than half that observed in the $\mathrm{Cre}^{-}$mice, revealing that most but not all of the air space IgG recognizing Sp3 required PD-L2 ${ }^{+}$B cells. Levels of pneumococcus-specific BALF IgA and IgM were also lower in $\mathrm{Cre}^{+}$mice, though these differences were less pronounced and did not reach statistical significance (Figure 8J). Some of the residual heterotypic antibody was likely that produced by lung plasma cells (Figure 3 ). We interpret these data as evidence that BRM cells contribute to lung antibacterial immunity, likely via the local secretion of heterotypic antipneumococcal IgG following reactivation.

\section{Discussion}

These studies show that mouse lungs recovered from pneumococcal infections contain BRM cells that contribute to serotypeindependent antipneumococcal protection. In light of these results - combined with the evidence that influenza also elicits lung BRM cells $(8,10,22)$ and our finding that normal human lung tissue is enriched with BRM-like cells - we propose that BRM cells are a common component of the lung adaptive immune cell repertoire.

We consistently observed lung B cells in clusters within the loose interstitial tissue of bronchovascular bundles, usually closer to the airways than the arteries, and often but not always with the concurrent presence of $\mathrm{CD}^{+} \mathrm{T}$ cells. However, we did not find evidence of tertiary lymphoid tissues in these pneumococcus-recovered lungs, which lacked HEVs and tissue-distorting lymphocyte aggregates on H\&E staining. This differs from influenza, which elicits these features in addition to lung BRM cells $(8,11)$. The tendency to be associated with areas of iBALT varies among the distinct populations of lung TRM cells in influenza-recovered mouse lungs (40). However, B cell studies in the context of influenza have not reported lung B cells outside of iBALT (40-42), and it is therefore unclear whether influenza-generated lung BRM cells reside only in these structures. The current pneumococcus studies dissociate BRM cells from localization exclusively in iBALT and reveal that lung BRM cells can be components of histologically unremarkable lungs.

In addition to our studies of pneumococcus-experienced mice, we observed cells with a BRM phenotype in disease-free human lung tissues, which had likely been exposed to both pneumococcus and influenza in addition to a wide variety of other respiratory pathogens. Fifty to seventy percent of human lung B cells in this study expressed the memory marker CD27, while the majority of $B$ cells typically observed in the blood of adult humans are naive (43). We conclude that the antigen-experienced lung represents a site of BRM cell accumulation across species.

We chose to give mice initial pneumococcal exposures directly in the lung, as opposed to using a colonization model. Whether the outcome after repeat i.t. exposures in mice truly mimics the lungs of humans, who are frequently colonized and less frequently experience true pneumonia events, may be questioned. However, there is evidence that colonization alone can change BALF macrophage and $\mathrm{T}$ cell populations in humans, and the density of pneumococcal colonization has been shown to correlate with the amount of pneumococcal DNA found in human $\operatorname{BALF}(44,45)$. These findings lead us to believe that the repeated colonization events experienced by most humans likely result in changes to the lung immune cell environment similar to those elicited by lobar exposures in mice.

Epidemiological evidence suggests that natural human exposures to pneumococcus confer immunity against both colonization and invasive disease by all pneumococcal serotypes, even those that are not common colonizers of children $(19,20)$. These observations indicate that unlike vaccine-induced pneumococcal immunity, natural antipneumococcal immunity is not directed against the serotype-specific pneumococcal capsular polysaccharide but instead targets conserved pneumococcal antigens. Many pneumococcal peptides and proteins are immunogenic and sufficient to induce B 
cell-dependent and $\mathrm{CD} 4^{+} \mathrm{T}$ cell-dependent immunity that is independent of serotype (46-48), so the heterotypic protection after recovery from pneumococcal pneumonia likely involves a panoply of antigen specificities. The difficulties in scaling up current serotype-specific pneumococcal vaccines to cover all serotypes has led to extensive interest in understanding what immune components are required for this natural heterotypic protection. Studies addressing this in mice have highlighted roles for lung and nasal TRM cells, remodeled alveolar macrophages, plasma antibodies, and bone marrow LLPCs $(20,24)$. A role for MBCs in serotype-independent immunity has not to our knowledge been previously investigated. The PZTD mouse model afforded us the opportunity to assess the effects of inducible depletion of memory marked (PD-L2+) B cells while leaving naive $\mathrm{B}$ cells and preexisting antibodies intact. Depletion of $\mathrm{CD} 19^{+} \mathrm{PD}-\mathrm{L} 2^{+} \mathrm{B}$ cells in pneumococcus-experienced mice led to a substantial loss of serotype-independent lung protection.

This result differs from those of a prior study in which pneumococcal colonization of mice protected against serotype-matched lung infection. In that setting, while LLPCs are crucial, MBCs are dispensable (27). These findings may reflect the particular importance of MBCs in responses to related but not identical infections. This partitioning of function has been observed for influenza and West Nile viruses, for which preexisting antibodies protect against homologous virus rechallenge, while reactivated MBCs confer immunity against an antigenically distinct virus $(21,22)$. We postulate that MBCs, especially those in the lung, also play an important role in the heterotypic immune response to bacterial respiratory pathogens. This would imply that vaccines against pathogens that rapidly mutate or comprise many subtypes should be designed with a goal of eliciting B cell memory in addition to circulating antibody.

A limitation to our studies is the difficulty in distinguishing the functional contributions of BRM cells from those of other B cell subpopulations, notably LLPCs, marginal zone B cells, and B 1 B cells. While the heterotypic IgM in the blood of experienced mice derives in part from splenic B cells, neither the heterotypic IgG in the blood nor the effective immune defense of the lung required $B$ cells in the spleen. We observed plasma cell establishment in both the bone marrow and lung following our pneumococcal infections. Bone marrow plasma cells are likely sources for heterotypic IgG in the blood, and lung plasma cells for heterotypic antibodies in the pulmonary air spaces. Although a full characterization of the relative contribution of each of these plasma cell populations to pneumococcal immunity is outside the scope of this work, our pre-opsonization study indicates that preformed plasma antibodies can contribute to pneumococcal control. The superior protection after lobar pneumonias observed in the ipsilateral compared with contralateral lobes (24) indicates that such circulating antibodies are insufficient to account for pneumonia prevention after recovery from prior infections.

In addition to lung BRM cells, many $\mathrm{B} 1 \mathrm{~B}$ cells are $\mathrm{PD}-\mathrm{L} 2^{+}$, and the pleural fluid is rich in B $1 \mathrm{~B}$ cells, which can migrate to the lung in response to pneumococcal exposures (35). We considered that DT-induced depletion of pleural fluid PD-L2 ${ }^{+} \mathrm{B} 1$ cells may contribute to the phenotype observed in the PZTD model. However, there are no substantial differences in the pleural fluid B cell compartment after pneumococcal experience, and we observe no change in the percentage of lung B $1 \mathrm{~B}$ cells upon Sp3 infection of experienced or naive $\mathrm{B} 6$ mice. Therefore, we conclude that the best explanation for our results is the loss of PD-L2 ${ }^{+}$lung BRM cells in DT-treated $\mathrm{Cre}^{+}$PZTD mice. The infection-stimulated elevation in heterotypic IgG in experienced lungs depends largely on PD-L2 ${ }^{+} \mathrm{B}$ cells. In contrast, Th17 and Th1 cells in experienced lungs were uncompromised by lack of $\mathrm{B}$ cells or depletion of $\mathrm{PD}-\mathrm{L} 2^{+} \mathrm{B}$ cells. We conclude that local antibody production is more likely than antigen presentation to be the mechanism of protection provided by lung BRM cells. These and other reported observations suggest a model of serotype-independent pneumococcal lung immunity involving early contributions of remodeled innate immune cells (24), TRM cells $(23,25)$, and preexisting antibodies; followed by a later, but crucial, contribution from lung BRM cells differentiating to secrete additional antipneumococcal antibodies.

The COVID-19 pandemic raises additional relevant questions. People who have recently recovered from respiratory infections by endemic human coronaviruses have less-severe COVID-19 outcomes (49), suggesting naturally acquired heterotypic immune protection against SARS-CoV-2. With regard to the evidence presented here that lung BRM cells are essential components of immunity against heterotypic respiratory infection, it will be important to test whether human lungs contain cross-reactive BRM cells elicited by the endemic human coronaviruses and, if so, whether those BRM cells influence the outcome of SARS-CoV-2 infection.

Many aspects of BRM cells demand further investigation, including factors leading to their recruitment and retention; their immunoglobulin repertoire; and whether BRM cells, like TRM cells, are found across a wide range of organ systems in mice and humans. Despite these unknowns, a burgeoning body of evidence including the present results have begun to shed light on the previously unrecognized importance of BRM cells in the lung. Our findings lead to a more complete picture of the natural lung defenses that pneumococcal exposures generate and are crucial for subsequent pneumonia protection. Defining these protective mechanisms in healthy hosts would guide studies into what aspects of this protection are lost with age or comorbidities. As many mucosal vaccine development strategies have begun to focus on eliciting resident memory cells, this knowledge will also be crucial for future investigations into correlates of optimal mucosal immunity.

\section{Methods}

Mouse experiments. B6 mice were purchased from the Jackson Laboratory. Ighm ${ }^{\text {tmlcgn }}(\mu \mathrm{MT})$ B cell-deficient mice on a B6 background were purchased from the Jackson Laboratory and maintained by breeding of homozygous animals. Homozygous PZTD (PD-L2 ${ }^{+}$indicator knockin and inducible knockout) mice with CD19-Cre transgenes were previously described (38). Mouse experiments were initiated when mice were 6-12 weeks of age and included both male and female animals. Mice were maintained in SPF conditions on a 12-hour light/12-hour dark cycle with ad libitum food and water. In all mouse experiments, groups included a minimum of 3 mice and were assessed in at least 2 separate experiments performed on different days unless otherwise indicated, and no outliers were excluded.

Bacterial infections. Antipneumococcal immunity was generated as previously described (23) by giving mice 2 exposures to $S$. pneumoniae serotype 19F (Sp19F EF3030; provided by Marc Lipsitch, Harvard University, Boston, Massachusetts, USA) 1 week apart before allowing mice to recover for at least 4 weeks. Sp19F exposures $\left(1 \times 10^{6}\right.$ bacterial CFU 
in $50 \mu \mathrm{L}$ ) were given either i.t. in the left lung lobe or i.n. (for Figure $3 \mathrm{H}$, Figure 6I, and Figure 8J). Challenge infections were given by instillation of $0.5 \times 10^{6}$ to $1 \times 10^{6} \mathrm{~S}$. pneumoniae serotype 3 (ATCC, 6303) i.t. or i.n. (for Figure 7D only). Bacterial burdens were assessed by counting bacterial CFU in plated whole lung homogenate.

Tissue collection. Samples were collected from mice after euthanasia by isoflurane overdose. Pleural fluid was collected by washing the pleural space with PBS. For BALF samples, the trachea was cannulated with an 18-gauge angiocatheter (Becton Dickinson, 381444), and the lungs were lavaged with PBS. For collection of lung tissue protein, harvested lungs were homogenized in a bullet blender (Next Advance). For ex vivo analysis of mouse bone marrow cell supernatants, single-cell suspensions of cells flushed from isolated femurs and tibiae were plated at $4 \times 10^{6}$ cells per mL in CTL-Test B Medium (ImmunoSpot, Cellular Technology Ltd.). Cells were cultured at $5 \% \mathrm{CO}_{2}$ and $37^{\circ} \mathrm{C}$ for 15 hours.

For human samples, lung tissue (determined by a pathologist to be normal) from 7 patients undergoing surgical lung cancer resection at Boston Medical Center was immediately immersed in RPMI medium and transported to our laboratory on ice. Expression of IgD, IgM, CD38, and CD83 was assessed in only the last 5 samples, accounting for why only 5 points are shown on graphs involving these markers.

Lung digestion and flow cytometry. For flow cytometry and ex vivo stimulations, isolated lung lobes were digested with type II collagenase (Worthington Biochemical) as previously described (23). For human lungs, tissue was similarly processed into single-cell suspensions using $0.2 \mu \mathrm{g} / \mathrm{mL}$ type A collagenase (Worthington Biochemical) and $40 \mu \mathrm{g} / \mathrm{mL}$ DNase I.

To distinguish circulating from tissue cells, we injected mice i.v. with $2 \mu \mathrm{g}$ of the indicated fluorescent anti-CD45 antibody 3 minutes before sacrifice. Single-cell lung suspensions were prepared as above, with left and right lobes digested separately. Single-cell spleen suspensions were obtained by filtering mechanically disrupted organs through $70-\mu \mathrm{m}$ filters. All single-cell suspensions from mice or human samples were stained with specified fluorescent antibodies (Supplemental Table 1). After staining, all samples were resuspended in buffer containing 7-AAD (BD Biosciences) viability dye, except in experiments assessing intracellular expression of Blimp-1, for which the Zombie UV Fixable Viability Kit (BioLegend, 423107) was used. Intracellular staining was achieved using a Transcription Factor Staining Buffer Set (Thermo Fisher Scientific, 00-5523-00) according to the manufacturer's instructions. Stained cells were assessed on an LSR II (BD Biosciences) or Cytek Aurora (Cytek Biosciences). Data were analyzed using FlowJo v10 software (BD Biosciences).

Cell depletion. For circulating B cell depletion studies, experienced mice were treated once with an anti-CD20 monoclonal antibody (clone $5 \mathrm{D} 2$, Genentech) or a rat IgG2a isotype control antibody (BioXCell) i.p. $(100 \mu \mathrm{g})$ and i.n. $(100 \mu \mathrm{g})$. For depletion of PD-L2 ${ }^{+} \mathrm{B}$ cells, experienced PZTD mice were treated once with i.t. (50 ng in the left lung lobe) and i.p. (50 ng) DT (MilliporeSigma, d0564) in sterile saline. Vehicle-treated mice were given sterile saline alone. For $\mathrm{CD} 4^{+}$cell depletion studies, $\mu \mathrm{MT}$ mice were treated with an anti-CD4 monoclonal antibody (clone GK1.5) or a rat IgG2b isotype control antibody (both from BioXCell). Antibodies were diluted in sterile saline and administered i.p. $(500 \mu \mathrm{g})$ and i.n. (100 $\mu \mathrm{g}) 72$ and 24 hours prior to Sp3 challenge.

Splenectomy. Mice were anesthetized via i.p. injection of ketamine (75 mg/kg) and xylazine (5 mg/kg). After superficial disinfection, the spleen was accessed and excised through a small incision through the skin and abdominal wall, after which the incision was closed. Negative control mice experienced the same anesthesia, surgical access to the peritoneal cavity, and incision closure but without splenectomy. Mice recovered for 3 weeks prior to infection.

ELISAs. Pneumococcus-specific antibodies were measured in plasma, ex vivo culture supernatants, and BALF using a whole bacterial cell ELISA. Nunc MaxiSorp plates (Thermo Fisher Scientific) were coated with $1 \times 10^{7}$ CFU of the indicated bacteria. The acapsular pneumococcal strain used as capture antigen in the culture supernatant and BALF ELISAs was derived from a serotype 3 parent strain (isolate provided by Stephen Pelton, Boston University). HRP-conjugated detection antibodies were as follows: antitotal IgG (1:2000, R\&D Systems, HAF007), anti-IgG3 (1:10,000, Abcam, ab97260), anti-IgG1 (1:10,000, Abcam, ab97240), anti-IgG2b (1:10,000, Abcam, RRID: AB_10695945), anti-IgG2c (1:10,000, Abcam, ab97255), anti-IgA (1:2000, Thermo Fisher Scientific, RRID:AB_2533951), anti-IgM (1:2000, Thermo Fisher Scientific, RRID: AB_2533954). The optical density of each well was determined at $450 \mathrm{~nm}$.

IL-17 and IFN- $\gamma$ were measured in lung homogenates using a DuoSet IL-17 ELISA kit (R\&D Systems, DY421) and DuoSet IFN- $\gamma$ ELISA kit (R\&D Systems, DY485), respectively, according to the manufacturer's instructions.

Ex vivo B cell stimulation and ELISpots. Mice were given $2 \mu \mathrm{g}$ i.v. PE anti-CD45 (BioLegend, RRID: AB_312971) 3 minutes before sacrifice. Single-cell suspensions of lung and spleen tissue were procured. Cells in lung tissue that were not protected from the i.v. stain were selected out using an EasySep Mouse PE Positive Selection Kit (StemCell Technologies, 17666) according to the manufacturer's instructions. All cells were then cultured in CTL-Test B Medium containing resiquimod (R848), IL-2 (ImmunoSpot, CTL-mBPOLYS-200), and 1\% L-glutamine (Thermo Fischer Scientific) for stimulated samples; or medium with $1 \% \mathrm{~L}-$ glutamine alone for unstimulated controls. Cells were incubated for 4 days at $5 \% \mathrm{CO}_{2}$ and $37^{\circ} \mathrm{C}$. Supernatants were used for antibody analysis. Cells were used for ELISpot assays using the Immunospot Mouse IgG Single-Color ELISpot kit following the manufacturer's instructions. For plasma cell ELISpots, single-cell suspensions of EV lung cells were directly plated onto ELISpot plates in CTL-Test B Medium for 8-12 hours before plates were analyzed using the Immunospot Mouse IgG or IgA Single-Color ELISpot kits. Developed ELISpot plates were visualized and counted on a BioSpot S5 Macro Analyzer using the Immunospot software package (Cellular Technology Ltd.).

Plasma pre-opsonization. Sp3 was added to $500 \mu \mathrm{L}$ pooled plasma from mice that had received i.n. Sp19F or saline $4-8$ weeks prior until the mixture reached an optical density of 0.3 . The mixture was rotated for 1 hour at $4^{\circ} \mathrm{C}$. Bacterial pellets were then resuspended in $500 \mu \mathrm{L}$ sterile saline, then $50 \mu \mathrm{L}$ was instilled i.n. into each recipient naive mouse.

Whole blood RNA-Seq. RNA was extracted from facial vein blood using the Mouse RiboPure-Blood RNA Isolation Kit (Thermo Fisher Scientific, AM1951) according to the manufacturer's instructions. RNA was purified, and a cDNA library was generated using a TruSeq Stranded Total RNA Library Prep Globin (Illumina, 20020612). Single-end 50-base-pair read length sequencing was performed on an Illumina HiSeq 2500. Libraries were constructed, quantified, and sequenced by the Tufts University Core Facility in Genomics. Illumina FASTQ files were quality trimmed using FastQC and Trimmomatic 0.36 (50), and were then aligned to the GENCODE GRCm38.p5 build of the mouse genome (https://www.gencodegenes.org/) using STAR 2.5.3a (51). Only uniquely aligned reads, which accounted for $47 \%-56 \%$ of reads from the sample set, were used for downstream analysis. The relatively 
low proportion of uniquely aligned reads was due to high amounts of sequenced noncoding RNA species. Indexed BAM files were then converted to raw gene counts using HTSeq (52) and the GENCODE M15 gene annotation reference. The Bioconductor package DESeq2 was used for statistical evaluations of expression differences between naive and experienced mice. RNA sequencing files have been deposited in the NCBI's Gene Expression Omnibus database (GEO GSE167103).

Immunofluorescence and confocal microscopy. Lungs of euthanized mice were prepared for immunofluorescence by i.t. instillation of OCT (Tissue-Tek) to inflate the lungs, which were then embedded and flash frozen in OCT. Frozen blocks were cut into $8 \mu \mathrm{m}$ sections and were then stained with primary antibodies: rabbit anti-CD4 (1:500, Abcam, ab183685); rat anti-B220 (1:1000, BD Pharmingen, 553084); followed by secondary antibody staining with Alexa Fluor 488 donkey anti-rabbit (1:1000, Jackson ImmunoResearch Laboratories Inc., 711-546-152), Alexa Fluor 594 donkey anti-rat (1:1000, Jackson ImmunoResearch Laboratories Inc., 712-586-153), and DAPI. Slides were imaged with a Leica DM4 microscope and Leica DFC 7000T camera, and were acquired with Leica LAS X software. Images were captured using a $20 \times$ objective with a numerical aperture of 0.55 ; or a $40 \times$ objective with a numerical aperture of 0.8 .

For confocal imaging of HEV markers, $10 \mu \mathrm{m}$ frozen sections of murine lungs or mediastinal lymph nodes prepared as above were used. Staining and imaging were performed as previously described (53). The primary antibodies used were rat anti-PNAd (1:1000, Santa Cruz Biotechnology, sc-19602) and rabbit anti-CD34 (1:200, Abcam, ab81289), with a DAPI counterstain. Images were captured with FluoView acquisition software on an Olympus FluoView 10i scanning laser confocal microscope using a $10 \times$ objective, numerical aperture 0.4. Photomultiplier tube gain and laser power settings were such that no signal was visible on isotype control sections. All images were processed in Image (NIH)/Fiji using identical look-up table settings for all images of the same magnification.

Multiplex fluorescence immunohistochemistry and multispectral whole imaging. FFPE tissue blocks were sectioned to $5 \mu \mathrm{m}$ using a RM2255 rotary microtome (Leica), transferred to positively charged slides, deparaffinized in xylene, and dehydrated in graded ethanol. Tissue sections were stained with H\&E for histologic examination, while serially cut unstained slides were used for immunohistochemistry.

Fluorescence multiplex immunohistochemistry was performed using Opal tyramide signaling amplification technology (Akoya Biosciences) on a Ventana Discovery Ultra (Roche) tissue autostainer (optimization parameters in Supplemental Table 2). After development of each antigen, heat-induced antigen retrieval was applied. Following the final round of antigen development, slides were counterstained with DAPI and mounted with ProLong Gold Antifade Mountant (Thermo Fisher Scientific). Uninfected mouse lungs served as negative controls.

Fluorescently labeled slides were imaged using a Mantra 2.0 Quantitative Pathology Imaging System (Akoya Biosciences). To maximize signal/noise ratios, images were spectrally unmixed using a synthetic library specific for the Opal fluorophores used for each assay and for DAPI. An unstained mouse lung section was used to create an autofluorescence signature that was subsequently removed from whole-slide images using InForm software version 2.4.8 (Akoya Biosciences).
Statistics. Statistical analyses were performed using GraphPad Prism 8.0. Each graph represents at least 2 independent experiments. Unless otherwise indicated, each individual point on a graph corresponds to an individual animal/sample. Plots displaying data as individual points and summary data depicted as violin plots are shown with medians indicated. Other summarized data are shown as means with standard deviations. Mann-Whitney $U$ tests were used to assess comparisons of 2 groups, while 3 or more groups were compared using either a Kruskal-Wallis test with Dunn's multiple-comparison test or a 2-way ANOVA with a Holm-Šidák multiple-comparison test. In multiple-comparison testing, all possible comparisons were made unless otherwise indicated. The FDR was calculated to control for multiple comparisons in RNA-Seq data. Two-sided $P$ values were calculated in all cases. Values of $P$ or FDR $q$ less than 0.05 were considered to indicate statistically significant differences.

Study approval. All animal protocols used in this study were approved by the Boston University Institutional Animal Care and Use Committee. Human samples were collected under protocols approved by the Boston University institutional review committees, and written informed consent was obtained from all participants.

\section{Author contributions}

KAB, ATS, NSE, NMSS, JLB, XZ, and JPM conceptualized the study. KAB, NSE, ATS, NMSS, CL, EIA, IMCM, WNG, AMSB, and AG performed experiments. KAB, ATS, CL, EIA, NSE, and ACB designed and validated flow cytometry panels. KAB, NSE, and MRJ performed statistical analyses. ACB, JLB, XZ, HK, NAC, MRJ, LJQ, and JPM provided resources and funding. KAB, JPM, NSE, ATS, NMSS, AMSB, and MRJ wrote the manuscript and designed the figures.

\section{Acknowledgments}

This work was supported by NIH grants including R35 HL-135756, R33 HL-137081, R01 AI-115053, R01 HL-111459, R01 HL-136725, F31 HL-142199, F31 HL-147397, and T32 HL-007035. We thank Stephen Pelton for provision of the acapsular pneumococcal isolate; Tom Kepler for analyzing RNA-Seq data; and Marc Lipsitch for provision of the Sp19F pneumococcal strain. The human samples were provided by the Boston University-Boston Medical Center (BU-BMC) Biospecimen Archive for Research Core (BARC) and BU-BMC Cancer Center, and we especially appreciate the expert assistance of Cheryl Spencer in procuring relevant materials. We thank Genentech for their generous provision of the anti-CD20 antibody. Flow cytometry data generation was facilitated by the Flow Cytometry Core Facility at Boston University School of Medicine (BUSM). This work utilized a Ventana Discovery Ultra autostainer that was purchased with funding from NIH grant S10 OD026983 (PAR-18-600). Schematic figures were created with BioRender.com.

Address correspondence to: Joseph P. Mizgerd, 72 East Concord Street, Boston, Massachussetts 02118, USA. Phone: 617.358.1186; Email: jmizgerd@bu.edu. AMSB's present address is: Pfizer Inc., Cambridge, Massachusetts, USA.
1. Beura LK, et al. Normalizing the environment recapitulates adult human immune traits in laboratory mice. Nature. 2016;532(7600):512-516.

2. Sathaliyawala T, et al. Distribution and com- partmentalization of human circulating and tissue-resident memory $\mathrm{T}$ cell subsets. Immunity. 2013;38(1):187-197.

3. Masopust D, Soerens AG. Tissue-resident T cells and other resident leukocytes. Annu Rev Immunol. 2019;37:521-546.

4. Turner DL, et al. Lung niches for the generation and maintenance of tissue-resident memory $\mathrm{T}$ 
cells. Mucosal Immunol. 2014;7(3):501-510.

5. Reboldi A, Cyster JG. Peyer's patches: organizing $\mathrm{B}$-cell responses at the intestinal frontier. Immunol Rev. 2016;271(1):230-245.

6. Mahallawi WH, et al. Infection with 2009 H1N1 influenza virus primes for immunological memory in human nose-associated lymphoid tissue, offering cross-reactive immunity to H1N1 and avian H5N1 viruses. J Virol. 2013;87(10):5331-5339.

7. Oh JE, et al. Migrant memory B cells secrete luminal antibody in the vagina. Nature. 2019;571(7763):122-126.

8. Allie SR, et al. The establishment of resident memory $\mathrm{B}$ cells in the lung requires local antigen encounter. Nat Immunol. 2019;20(1):97-108.

9. Joo HM, et al. Broad dispersion and lung localization of virus-specific memory B cells induced by influenza pneumonia. Proc Natl Acad Sci U S A. 2008;105(9):3485-3490.

10. Onodera T, et al. Memory B cells in the lung participate in protective humoral immune responses to pulmonary influenza virus reinfection. Proc Natl Acad Sci U S A. 2012;109(7):2485-2490.

11. GeurtsvanKessel $\mathrm{CH}$, et al. Dendritic cells are crucial for maintenance of tertiary lymphoid structures in the lung of influenza virus-infected mice. JExp Med. 2009;206(11):2339-2349.

12. Silva-Sanchez A, Randall TD. Role of iBALT in respiratory immunity. Curr Top Microbiol Immunol. 2020;426:21-43.

13. Tschernig T, Pabst R. Bronchus-associated lymphoid tissue (BALT) is not present in the normal adult lung but in different diseases. Pathobiology. 2000;68(1):1-8.

14. Pichyangkul $\mathrm{S}$, et al. Tissue distribution of memory $\mathrm{T}$ and $\mathrm{B}$ cells in rhesus monkeys following influenza A infection. J Immunol. 2015;195(9):4378-4386.

15. Quinton LJ, Mizgerd JP. Dynamics of lung defense in pneumonia: resistance, resilience, and remodeling. Annu Rev Physiol. 2015;77:407-430.

16. Fry AM, et al. Trends in hospitalizations for pneumonia among persons aged 65 years or older in the United States, 1988-2002. JAMA. 2005;294(21):2712-2719.

17. Jain $S$, et al. Community-acquired pneumonia requiring hospitalization among U.S. adults. $N$ Engl J Med. 2015;373(5):415-427.

18. Zuccotti G, et al. Serotype distribution and antimicrobial susceptibilities of nasopharyngeal isolates of Streptococcus pneumoniae from healthy children in the 13-valent pneumococcal conjugate vaccine era. Vaccine. 2014;32(5):527-534.

19. Granat SM, et al. Epidemiological evidence for serotype-independent acquired immunity to pneumococcal carriage. J Infect Dis. 2009;200(1):99-106.

20. Ramos-Sevillano E, et al. Mechanisms of naturally acquired immunity to Streptococcus pneumoniae. Front Immunol. 2019;10:358.
21. Purtha WE, et al. Memory B cells, but not long-lived plasma cells, possess antigen specificities for viral escape mutants. J Exp Med. 2011;208(13):2599-2606.

22. Adachi $Y$, et al. Distinct germinal center selection at local sites shapes memory B cell response to viral escape. J Exp Med. 2015;212(10):1709-1723.

23. Smith NM, et al. Regionally compartmentalized resident memory $\mathrm{T}$ cells mediate naturally acquired protection against pneumococcal pneumonia. Mucosal Immunol. 2018;11(1):220-235.

24. Guillon A, et al. Pneumonia recovery reprograms the alveolar macrophage pool. JCI Insight. 2020;5(4):133042.

25. Shenoy AT, et al. Lung $\mathrm{CD} 4^{+}$resident memory $\mathrm{T}$ cells remodel epithelial responses to accelerate neutrophil recruitment during pneumonia. Mucosal Immunol. 2020;13(2):334-343.

26. Ridderstad A, Tarlinton DM. Kinetics of establishing the memory B cell population as revealed by CD38 expression. J Immunol. 1998;160(10):4688-4695.

27. Bou Ghanem EN, et al. Nasopharyngeal exposure to streptococcus pneumoniae induces extended age-dependent protection against pulmonary infection mediated by antibodies and CD138(+) cells. J Immunol. 2018;200(11):3739-3751.

28. Nutt SL, et al. BLIMP1 guides the fate of effector B and T cells. Nat Rev Immunol. 2007;7(12):923-927.

29. Chernova I, et al. Lasting antibody responses are mediated by a combination of newly formed and established bone marrow plasma cells drawn from clonally distinct precursors. JImmunol. 2014;193(10):4971-4979.

30. Montalvao F, et al. The mechanism of anti-CD20-mediated B cell depletion revealed by intravital imaging. J Clin Invest. 2013;123(12):5098-5103.

31. Purwar R, et al. Resident memory $\mathrm{T}$ cells $(\mathrm{T}(\mathrm{RM}))$ are abundant in human lung: diversity, function, and antigen specificity. PLoS One. 2011;6(1):e16245.

32. Arpin C, et al. Generation of memory B cells and plasma cells in vitro. Science. 1995;268(5211):720-722.

33. Li Z, et al. CD83: activation marker for antigen presenting cells and its therapeutic potential. Front Immunol. 2019;10:1312.

34. Tomayko MM, et al. Cutting edge: hierarchy of maturity of murine memory $\mathrm{B}$ cell subsets. JImmunol. 2010;185(12):7146-7150.

35. Weber GF, et al. Pleural innate response activator B cells protect against pneumonia via a GM-CSFIgM axis. J Exp Med. 2014;211(6):1243-1256.

36. Hondowicz BD, et al. IL-2 is required for the generation of viral-specific $\mathrm{CD} 4^{+}$Th1 tissueresident memory cells and $\mathrm{B}$ cells are essential for maintenance in the lung. Eur J Immunol. 2018;48(1):80-86.

37. Cerutti A, et al. Marginal zone B cells: virtues of innate-like antibody-producing lymphocytes. Nat Rev Immunol. 2013;13(2):118-132.

38. Lee RA, et al. Fluorescence tagging and inducible depletion of PD-L2-expressing B-1 B cells in vivo. Ann N Y Acad Sci. 2015;1362:77-85.

39. Adler LN, et al. The other function: class II-restricted antigen presentation by B cells. Front Immunol. 2017;8:319.

40. Swarnalekha N, et al. Redefining CD4 T cell residency: helper $\mathrm{T}$ cells orchestrate protective humoral immunity in the lung [preprint]. https:// doi.org/10.1101/2020.02.28.963280. Posted on bioRxiv February 28, 2020.

41. Tan HX, et al. Inducible bronchus-associated lymphoid tissues (iBALT) serve as sites of $B$ cell selection and maturation following influenza infection in mice. Front Immunol. 2019;10:611.

42. Son YM, et al. Tissue-resident $\mathrm{CD}_{4}{ }^{+} \mathrm{T}$ helper cells assist the development of protective respiratory $\mathrm{B}$ and $\mathrm{CD} 8^{+} \mathrm{T}$ cell memory responses. $\mathrm{Sci}$ Immunol. 2021;6(55):eabb6852.

43. Morbach H, et al. Reference values for B cell subpopulations from infancy to adulthood. Clin Exp Immunol. 2010;162(2):271-279.

44. Mitsi E, et al. Nasal pneumococcal density is associated with microaspiration and heightened human alveolar macrophage responsiveness to bacterial pathogens. Am J Respir Crit Care Med. 2020;201(3):335-347.

45. Wright AK, et al. Experimental human pneumococcal carriage augments IL-17A-dependent T-cell defence of the lung. PLoS Pathog. 2013;9(3):e1003274.

46. Wilson R, et al. Naturally acquired human immunity to pneumococcus is dependent on antibody to protein antigens. PLoS Pathog. 2017;13(1):e1006137.

47. Moffitt KL, et al. T(H)17-based vaccine design for prevention of Streptococcus pneumoniae colonization. Cell Host Microbe. 2011;9(2):158-165.

48. Giefing C, et al. Discovery of a novel class of highly conserved vaccine antigens using genomic scale antigenic fingerprinting of pneumococcus with human antibodies. J Exp Med. 2008;205(1):117-131.

49. Sagar M, et al. Recent endemic coronavirus infection is associated with less-severe COVID-19. JClin Invest. 2021;131(1):143380.

50. Bolger AM, et al. Trimmomatic: a flexible trimmer for Illumina sequence data. Bioinformatics. 2014;30(15):2114-2120.

51. Dobin A, et al. STAR: ultrafast universal RNA-seq aligner. Bioinformatics. 2013;29(1):15-21.

52. Anders S, Pyl PT, Huber W. HTSeq-a Python framework to work with high-throughput sequencing data. Bioinformatics. 2015;31(2):166-169.

53. Barron AMS, et al. Perivascular adventitial fibroblast specialization accompanies $T$ cell retention in the inflamed human dermis. JImmunol. 2019;202(1):56-68. 